\title{
Cognitive-Radio Systems for Spectrum, Location, and Environmental Awareness
}

\author{
Hasari Celebii ${ }^{1}$, Ismail Güvenç ${ }^{2}$, Sinan Gezici ${ }^{3}$, and Hüseyin Arslan ${ }^{4}$ \\ ${ }^{1}$ Department of Electrical and Computer Engineering \\ Texas A \& M University at Qatar \\ PO Box 23874 Texas A \& M Engineering Building Education City, Doha, Qatar \\ Tel: 974-4230455; E-mail: hasari.celebi@qatar.tamu.edu \\ ${ }^{2}$ Wireless Access Laboratory \\ DOCOMO USA Labs \\ 3240 Hillview Avenue, Palo Alto, CA 94304, USA \\ Tel: +1 (650) 496-4781; E-mail: iguvenc@docomolabs-usa.com \\ ${ }^{3}$ Department of Electrical and Electronics Engineering \\ Bilkent University \\ Bilkent, Ankara 06800, Turkey \\ Tel: +90-312-290-3139; E-mail: gezici@ee.bilkent.edu.tr \\ ${ }^{4}$ Electrical Engineering Department \\ University of South Florida \\ 4202 E. Fowler Ave., ENB-118, Tampa, FL 33620, USA \\ Tel: +1 (813) 974-3940; E-mail: arslan@eng.usf.edu
}

\begin{abstract}
In order to perform reliable communications, a system needs to have sufficient information about its operational environment, such as spectral resources and propagation characteristics. Cognitive-radio technology has capabilities for acquiring accurate spectrum, location, and environmental information, due to its unique features such as spectrum, location, and environmental awareness. The goal of this paper is to give a comprehensive review of the implementation of these concepts. In addition, the dynamic nature of cognitive-radio systems - including dynamic spectrum utilization, transmission, the propagation channel, and reception - is discussed, along with performance limits, challenges, mitigation techniques, and open issues. The capabilities of cognitive-radio systems for accurate characterization of operational environments are emphasized. These are crucial for efficient communications, localization, and radar systems.
\end{abstract}

Keywords: Cognitive radio; cognitive positioning system (CPS); cognitive radar; Cramer-Rao lower bound (CRLB); environment mapping; communication channels; propagation channel; spectrum sensing; seamless positioning

\section{Introduction}

$\mathbf{C}$ ognitive radio (CR) is a promising approach for addressing the problems of next-generation wireless systems [1]. Although a globally recognized and clear-cut definition of cognitive radio does not yet exist [2], there are significant efforts towards this goal, such as the formation of the IEEE Standards Coordinating Committee 41 [3]. One of the main objectives of this committee is to technically define recent terminologies, such as cognitive radio, software-defined radio, and spectrum-agile radio. In this paper, we adopt a definition that includes the majority of the features of cognitive-radio systems reported in the literature [4]: sensing, awareness, learning, decision, adaptation, reconfigurability, and goal-driven and autonomous operation. It is apparent from the definition that cognitive radio is typically envisioned to have very sophisticated human-like features [5].
Relying on the fact that spectrum awareness (i.e., sensing, shaping, learning, and optimization) is one of the most crucial features of cognitive-radio systems, a significant portion of the studies in the literature focused on spectrum awareness and related issues [6-12]. Energy detectors, autocorrelation detectors, and cyclic autocorrelation detectors are three common techniques in the literature for detection of spectrum opportunities [13, 14]. Various approaches for spectrum sensing in cognitive-radio systems have been proposed in the literature. For instance, different single-antenna spectrum-sensing techniques were proposed in [1525]. In order to further improve the performance of spectrum sensing, cooperative spectrum-sensing techniques have been developed [26-33]. In addition, multiple-antenna spectrum-sensing methods, such as those in [34-38] were proposed to exploit spacediversity gain in spectrum-aware systems. More specifically, a spectrum-sensing technique based on an energy-detector receiver 
and multiple antennas was proposed in [35]. Finally, we refer the readers to $[7,8,10-12]$ for comprehensive surveys on spectrum awareness for cognitive-radio systems.

Unlike the spectrum-awareness feature, the other features of cognitive-radio systems, such as location and environmental awareness, have not been thoroughly investigated in the literature. The main milestones towards embodying location and environmental-awareness features in cognitive-radio systems can be summarized as follows. Haykin introduced the concept of cognitive radar, which is a way of learning about the surrounding environment $[39,40]$. In [39], a cognitive radar model that used an intelligent illuminator on the transmitter side and a radar-scene analyzer and Bayesian target-tracker methods on the receiver side was proposed. This was followed by the introduction of a radio-environment mapping method for cognitive-radio networks [41]. A joint sequential hypothesis testing and adaptive waveform method for target recognition in cognitive radar was proposed [42]. A conceptual framework of an environmental-awareness engine for cognitive-radio systems, along with its main functionalities, were proposed in [5].

On the other hand, the previous work on location awareness can be summarized as follows. A conceptual framework for the location-awareness engine of cognitive-radio systems was proposed in [43]. Furthermore, a cognitive positioning system (CPS), which is a step towards realization of cognitive location sensing, was introduced in [2, 44]. In [2], the range-accuracy adaptation feature of a cognitive positioning system was introduced without providing its performance analysis. Furthermore, in [45], a highprecision ranging algorithm for dynamic-spectrum-access networks was proposed. Theoretical limits on ranging for cognitiveradio localization systems in the presence of interference were studied, and an optimal spectrum-allocation strategy that provides the best ranging accuracy was proposed in [46]. The fundamental limits of time-delay estimation in dispersed-spectrum cognitiveradio systems were investigated in [47]. In that study, the CramerRao lower bounds for known and unknown carrier-frequency offset (CFO) were derived, and the effects of the number of available dispersed bands and modulation schemes were investigated. Also, [48] considered time-delay estimation in dispersed-spectrum cognitive-radio systems, and proposed two-step approaches for utilizing the frequency diversity in such systems. In addition, the performance comparison of whole and dispersed-spectrum utilization methods for cognitive-radio systems was studied in the context of time-delay estimation in [49]. Finally, the idea of seamless positioning as a functionality of the location-awareness engine for cognitive-radio systems was introduced in $[43,5]$ without proposing a specific technique.

In the literature, there exist studies that combine multiple different concepts for cognitive-radio systems. For instance, a conceptual model that combines location and environmental-awareness concepts was proposed by Yarkan et. al. [50], in order to improve the performance of cognitive-radio systems. Haykin introduced the concept of cognitive dynamic systems, in which cognitive radio for communications and cognitive radar for remote sensing were unified [51]. In addition, a unified framework that combines cognitive radio and cognitive radar was proposed in [52]. The concepts of localization and environmental mapping were merged, which is known as the simultaneous localization and mapping (SLAM) problem in the literature [53, 54]. In addition, in [5557], an ultra-wideband- (UWB) based simultaneous localization and mapping technique for mobile nodes was proposed for simple two-wall and four-wall scenarios. In that context, a comprehensive unified cognitive-radio-system model that combined spectrum, environmental, and location awareness concepts was proposed at a conceptual level in [5]. Note that [5] introduced that comprehensive conceptual model without providing the details of how it could be implemented and designed. Therefore, in this paper, we extend the study in [5] by examining the critical implementation issues that need to be addressed for this model in order to move it from concept to reality. In addition, the challenges and efforts for the deployment of this system model are presented in this study.

The main focus of this study is to provide an in-depth discussion and overview of challenges, efforts, limitations, and implementation issues for the realization of a cognitive-radio-system model with spectrum, environmental, and location-awareness capabilities. The paper is organized as follows. The overall system architecture for cognitive-radio systems with spectrum, location, and environmental-awareness capabilities is summarized in Section 2. Section 3 is a brief review of spectrum-aware systems, especially spectrum sensing and utilization techniques, and spectrum regulations. In Section 4, a discussion of location-aware systems, along with cognitive positioning systems and seamless positioning systems, is presented. In Section 5, the details of environmentally aware systems are provided by emphasizing cognitiveradar and topographical information-estimation functionalities. In Section 6, the dynamic operation of cognitive-radio systems, along with challenges, adaptation techniques, and open issues, are discussed in the context of a cognitive-positioning system. Finally, the concluding remarks are presented in Section 7.

\section{System Model}

The cognitive-radio architecture [5] in Figure 1 is considered to be the system model in this paper. Goal-driven and autonomous operation are two main features distinguishing cognitive radios from legacy radios. Cognitive radios achieve goals by determining

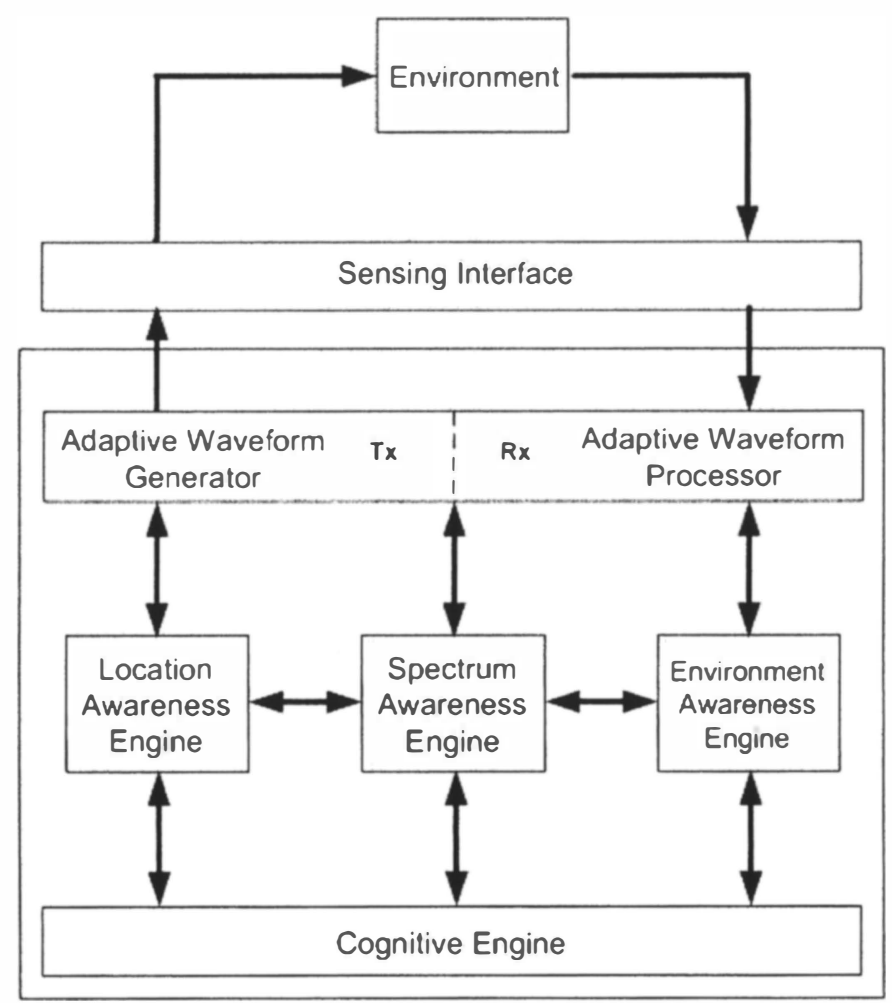

Figure 1. A simplified block diagram of the proposed cognitiveradio-system model. 
the appropriate radio parameters for the perceived, current, dynamic wireless-channel environmental conditions [58]. The goals can be network and node-level goals [59]. Since the focus of this paper is cognitive-radio nodes, node-level goals are considered. In addition, a goal can consist of single or multiple objectives. For example, minimization of bit-error-rate (BER), maximization of throughput, and minimization of power consumption are three examples of cognitive-radio objectives [58]. These objectives can be achieved by using artificial-intelligence (AI) methods, such as genetic algorithms. For instance, a cognitive engine based on a multiple-objective fitness function using a simple weighted-sum method was proposed in [58] for the achievement of cognitiveradio goals. That method was capable of instantly switching operating goals by simply modifying the objective weighting factor in dynamic wireless-channel environments.

Goal-driven and autonomous operations are managed by the cognitive engine in the system model shown in Figure 1. In other words, the cognitive engine maps the final goals to the local goals, and assigns the local goals to the respective specialized engines. Finally, the cognitive engine collects and combines the results of the assigned local goals to achieve the final goals. Note that the cognitive-radio-system model can have many engines, such as policy, interference, and topology engines; however, in this study, we limit the system model to have three engines, which are the spectrum, location, and environmental-awareness engines. The main functionalities of the spectrum, location, and environmentalawareness engines are to handle all the tasks related to the spectrum, location, and environmental information of the cognitive radio, respectively. For instance, the spectrum-awareness engine is responsible for performing spectrum-information-related tasks, such as estimation of available bands, carrier frequencies, and bandwidths. Similarly, the environmental-awareness engine is responsible for handling environmental-information-related tasks, such as recognition of objects in the surrounding environment. Note that the environmental definition proposed in [5] is employed in this study. This is defined with the following entities: topographical information, object information, propagation-channel characteristics, and meteorological information. Likewise, the location-awareness engine is responsible for handling locationinformation-related tasks, such as range and position estimation. Once the cognitive engine determines the radio parameters for achieving a final goal, it then adapts the radio parameters using the arbitrary-waveform generator/processor, as well as the sensing interface in the system model [5]. The arbitrary-waveform generator/processor is an interface that can generate and process any type of waveform at the transmitter/receiver side, respectively. The sensing interface consists of different sensors, such as radiosensing, radio-vision, and radio-hearing devices, to send/acquire signals to/from the environment [5].

Software-defined radio (SDR) is a key enabling technology for deploying cognitive-radio systems such as shown in Figure 1. All the engines can be implemented in microprocessors [60]. However, implementing such sophisticated algorithms in microprocessors can require high power consumption, and can generate an excessive amount of heat. Therefore, low-complexity spectrum, location, and environmentally aware algorithms need to be developed to in order to address these two issues. In addition, small-size and low-power cooling systems can be designed. Adaptive-waveform generation and processing include performing baseband and $\mathrm{RF}$ operations in the transmitter and receiver, respectively, in an adaptive manner. Adaptive-waveform generation and processing functionalities can be deployed using reconfigurable digital-radio processors, such as FPGAs (field-programmable gate arrays) and DSPs (digital signal processors) [60]. The evaluation and comparison of reconfigurable digital-radio technologies for cognitive-radio systems was studied in [60]. One of the main challenges in implementing baseband and RF functionalities is the stage of digitizing the signal. In an ideal software-defined radio approach, the RF signal is digitized right after the antenna, and then the digital RF signal is processed using reconfigurable digital processors. Two main challenges to implementing such architectures are designing highperformance data converters that sample the RF signal at multigigasamples/second (Gsps) rates, and high-performance digitalradio processors that run at tens of Gbps data rates. Similarly to general-purpose microprocessors, reconfigurable digital-radio processors have high-power-consumption and overheating problems. In the second approach, adaptive-waveform generation and processing is implemented using mixed digital and analog circuitries. In such an approach, the signal is digitized at the IF stage, and the digital IF signal is processed by reconfigurable digital-radio processors. This relaxes the requirements of the data converters and reconfigurable digital-radio processors. On the other hand, this approach requires the use of software-tunable RF components, such as software-tunable filters, power amplifiers, and up/downconverters. Additionally, high-performance software-tunable power circuitry, clock-generation circuitry, frequency synthesizers, and impedance-matching synthesizers are required. The further details of the requirements and challenges of implementing adaptive-waveform generation and processing can be found in [60].

The sensing interface consists of different sensors, such as an antenna, a camera, and an acoustic sensor. The most extensively used air-sensing interface is an antenna, which is the main focus in this study. There are significant efforts towards the development and design of software-tunable antenna systems and RF front-ends for software-defined radios. For instance, a reconfigurable antenna was proposed in [61] that can be tuned electronically to different frequency bands while satisfying both high-efficiency and narrowinstantaneous-bandwidth requirements. In addition, a novel FPGAbased RF front-end architecture was proposed in [61]. The proposed novel RF front-end, coupled with the reconfigurable antenna, is a promising architecture for realizing software-defined radio.

Advanced, comprehensive, and intelligent applications can be supported by the system model in Figure 1. For instance, consider a scenario in which a user is employing a cognitive radio in a car, and is moving towards a harsh environment, such as a tunnel in an urban area. Assume that the cognitive-radio user operates in the normal mode before entering to the tunnel. In the normal mode, the main goal is to achieve the best throughput possible while satisfying a power constraint. In addition, it is assumed that a high carrier frequency is used, since penetration is not a major problem in the normal mode. We call the operating mode within the tunnel the alert mode. This mode is defined as the mode in which the main goal is to keep the level of the quality of service (QoS) as close as possible to the quality-of-service level provided outside the tunnel. The location-awareness engine tracks the cognitiveradio user in order to provide its position information. On the other hand, the environmental-awareness engine acquires the channel environment information within and outside the tunnel, such as the propagation model (path-loss coefficient). Furthermore, the spectrum-awareness engine provides the available bands within and outside the tunnel. The location-awareness engine detects when the cognitive-radio user enters the tunnel, and then the cognitive engine switches the operating mode from the normal to the alert mode. Since signal penetration and propagation are major issues for the tunnel environment, the cognitive engine lowers the carrier frequency and maximizes the transmitted power, in order to improve the penetration. In addition, it adapts the coding and modulation to combat the fading. Once the location-awareness engine detects that the cognitive-radio user has exited the tunnel, 
the cognitive engine then switches the mode back to the normal mode. Note that numerous goal-driven and autonomous applications can be supported by the system model. Of course, such intelligence comes with additional complexity. However, recent advances in software-defined radio technology, mobile computing, and processors, such as sophisticated FPGAs, are promising for implementing such system models in the near future [60]. There are significant efforts from academia, industry, and regulatory agencies in the development and design of cognitive-radio systems. For instance, a real-time MIMO OFDM (orthogonal frequency-domain multiplex) test bed for cognitive radio networks was developed in [62], and Mishra et. al. developed a real-time cognitive-radio test bed for the physical and link layers. In addition, a biologically inspired cognitive-radio test bed was developed in $[63,64]$, in order to develop and test genetic algorithms for cognitive-radio systems.

\section{Spectrum-Aware Systems}

Dynamic spectrum utilization is an essential component of cognitive-radio and cognitive-positioning systems. As illustrated in Figure 1, spectrum sensing is handled through the spectrumawareness engine in a cognitive-radio system. Its output can be sent to cognitive, location, and environmental-awareness engines. A spectrum-awareness engine may also utilize the information from the other engines in order to improve its own accuracy.

As shown by numerous studies in the literature (see, e.g., [612]), the spectrum is utilized inefficiently by today's wireless networks. In many scenarios, certain parts of the spectrum are not utilized by any user, which is a waste of spectral resources. In some other scenarios, the interference from other users may be negligible, due to propagation characteristics and signal attenuation. In such case, some secondary users can have the opportunity to re-use certain parts of the spectrum. However, such opportunistic spectrum utilization requires reliable spectrum sensing to make sure that the frequency band of interest is not occupied by any primary users in a given time interval. If the spectrum is available, it may then be utilized by an opportunistic network for communications and/or localization purposes.

An example of an opportunistic spectrum-utilization approach is illustrated in Figure 2, where several primary users (PUs) are communicating with a base station (BS). Moreover, there is a secondary network ( $\mathrm{SN}$ ), which tries to utilize the spectral resources of the primary network. The signals of the primary users that are far away from the secondary network are attenuated significantly, and hence their uplink (UL) spectrum may be available for reuse in certain regions of the secondary network. For example, SU-2 may reuse the spectrum of PU-4, since the received signal power from PU-4 is very weak at SU-2.

\subsection{Spectrum Sensing}

Spectrum sensing is one of the most fundamental problems for cognitive radios. Two main issues in spectrum sensing are reliability and wideband sensing [65]. Reliable detection of the existence of primary users is a primary requirement for the minimization of interference to existing communications. Note that a hidden node is major threat for reliable primary-user detection, where a secondary user cannot detect the primary user's signals due to shadowing. We will briefly discus two main approaches that improve the reliability of spectrum sensing: collaboration, and multi-antenna-based spectrum-sensing methods.

Spectrum-sensing techniques for opportunistic spectrum access have been investigated extensively in the literature. Several single-antenna spectrum-sensing techniques were proposed in [15$25]$. As discussed in $[13,14]$, there are three common techniques for detection of spectrum opportunities: energy detectors, autocorrelation detectors, and cyclic autocorrelation detectors. As illustrated in Figure 2, an energy-detector receiver at SU-2 is a common spectrum-sensing method for determining the spectrum opportunities. An energy detector sets a threshold, and the frequency bands with received power below the threshold are labeled as spectrum opportunities. The spectrum-sensing results may be further improved through cooperative techniques, as in [26-33], where multiple closely located receivers exchange information for more-accurately determining spectrum opportunities.

Multiple-antenna spectrum sensing [34-38] is a second alternative for improving the reliability of spectrum-sensing results. In Figure 3, spectrum sensing based on an energy-detector receiver and multiple antennas is illustrated [35]. After the signals arriving at different antennas are processed with a bank of squarelaw devices and integrators, the resulting decision variables are

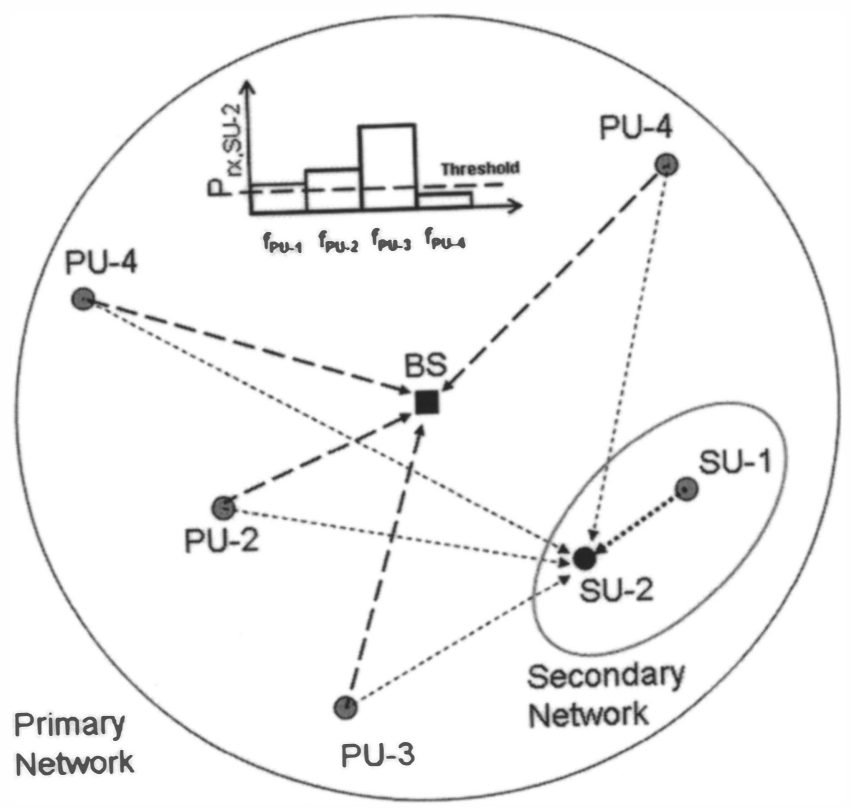

Figure 2. An illustration of spectrum opportunities in a secondary network that arise due to propagation effects in a primary network. $P_{r x ; S U-2}$ and $f_{P U-i}$ denote the received signal energy at SU-2 and the frequency band for primary user $i$, respectivelv.

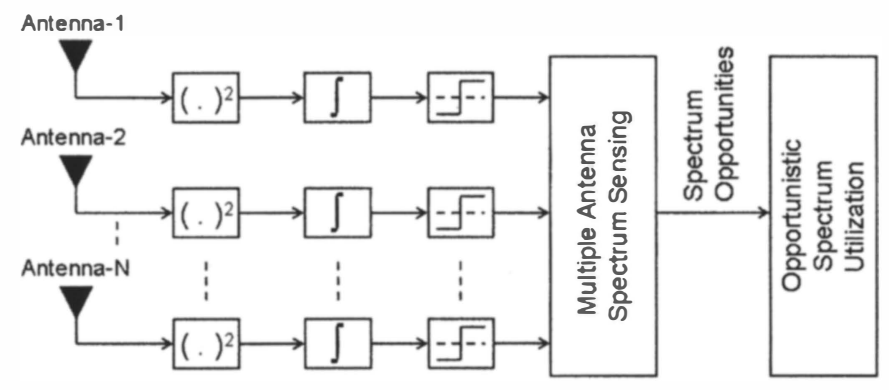

Figure 3. Multiple-antenna spectrum sensing at a cognitiveradio receiver [35]. 
compared with a threshold, followed by a multi-antenna spectrumsensing block. For example, in [35], binary spectrum-sensing decisions were made corresponding to each of the antennas. The number of positive hypotheses (corresponding to the decision that the spectrum is occupied) were then compared with a threshold. If this was larger than the threshold, the spectrum was occupied, and otherwise, it was available. On the other hand, [34] coherently combined the multiple-antenna signals, without any prior information about the primary system, and fed the spectral-correlation function of the combined signal to a feature detector for the final decision. The results in [34] and [35] showed considerable improvement when using multiple antennas over the single-antenna spectrumsensing technique.

Another main challenge in spectrum sensing is to sense very wide bandwidths, up to several $\mathrm{GHz}$, in real time, in order to reliably detect available bands for opportunistic usage [65]. It is a challenging task to develop and design an RF front-end and digitalsignal-processing algorithms that satisfy such sensing requirements. Along this line, a multi-band joint-detection method was proposed in [65] for achieving wideband spectrum sensing.

\subsection{Utilization of Spectrum Opportunities}

After sensing the spectrum and determining the available frequency bands, a cognitive-radio system needs to efficiently utilize these-spectral resources in order to perform reliable communications [9], localization [2, 5], and/or radar [39] functions. In utilizing available spectral resources, the main criteria to consider are performance, complexity, and power consumption.

In communications systems, performance can be quantified via throughput, whereas in localization systems, it is related to average position-estimation error $[66,67]$. On the other hand, the performance of radar systems is characterized by probabilities of detection and false alarm, as well as accuracy of velocity and position estimates (time and Doppler resolution) related to targets [68, 69]. In the aforementioned systems, performance improvements can be obtained, such as an increase in system bandwidth and/or signal-to-interference-plus-noise (SINR). The main implication of improved performance with increased bandwidth is to utilize all of the available bands in the spectrum. For example, if a scenario such as in Figure 4 is considered, the best theoretical performance can be obtained when all the available bands around the center frequencies $f_{c_{1}}, \ldots, f_{c_{K}}$ are used. However, in practical scenarios, utilization of many dispersed bands, such as in Figure 4, can considerably increase the complexity of the system. For example, simultaneous processing of signals that occupy various dispersed bands may require antennas and RF components that operate on extremely large bandwidths, which are difficult to design $[67,70]$. In addition to increasing complexity, the use of many dispersed frequency bands can significantly increase power consumption, which is not suitable for battery-operated applications. A suitable selection of the available bands and associated power levels should therefore be determined in order to meet performance, complexity, and cost requirements.

The center frequencies of the bands can also be important, in addition to their bandwidths, in selecting an operating spectrum from available bands. As the center frequency increases, more attenuation is expected for the signal occupying that band. Coverage of a system that employs available bands around high carrier frequencies can therefore be reduced, since signal power at a given distance decreases in such a case.

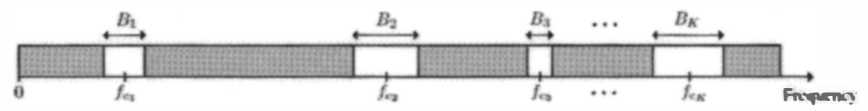

Figure 4. An example scenario illustrating available (white) and unavailable (gray) frequency bands.

After a cognitive-radio system determines which spectrum to utilize, the next important issue is to efficiently utilize that selected spectrum. Since there is a limit on the amount of transmitted power, the allocation of the signal power among different frequency bands, and inside different frequency components in each band, should be optimized in order to maximize system performance. For example, water-filling algorithms can be used to maximize the capacity of a communications system [71, 72]. Similarly, power can be optimally distributed among available frequency components in order to improve accuracy of a ranging system [46]. Theoretical limits on range-estimation accuracy for dispersed spectrum utilization were also studied in [47]. More details on [47] are presented in Section 4.

\subsection{FCC Regulations and Experimental Studies}

The Federal Communications Commission (FCC) in the United States has recently announced rules that allow unlicensed devices to communicate in the broadcast television (TV) spectrum, where the spectrum is not used by licensed devices [73]. Even though this unused TV spectrum (commonly referred to as "white spaces") presents a significant amount of spectrum to be used by cognitive-radio devices, the FCC provides a set of conservative rules and a framework for the operation of such devices. Some representative restrictions from the FCC's Second Report and Order on the operation of white-space devices are as follows [73]:

- “All devices, except personal/portable devices operating in client mode, must include a geolocation capability and provisions to access over the Internet a database of protected radio services and the locations and channels that may be used by the unlicensed devices at each location. The unlicensed devices must first access the database to obtain a list of the permitted channels before operating."

- $\quad$ "Fixed and personal/portable devices must also have a capability to sense TV broadcasting and wireless microphone signals as a further means to minimize potential interference. However, for TV broadcasting the database will be the controlling mechanism."

- "Devices must adhere to certain rules to further mitigate the potential interference and to help remedy potential interference should it occur. For example, all fixed devices must register their locations in the database. In addition, fixed devices must transmit identifying information to make it easier to identify them if they are found to interfere."

- "All white space devices are subject to equipment certification by the FCC Laboratory. The Laboratory will request samples of the devices for testing to ensure that they meet all the pertinent requirements." 
- "The Commission will act promptly to remove any equipment found to be causing harmful interference from the market and will require the responsible parties to take appropriate actions to remedy any interference that may occur."

Several cognitive-radio prototypes and related experimental work have been reported in the literature. For example, the FCC documented a comprehensive report on the performance evaluation of prototype white-space devices in [74]. Five devices (provided by Adaptrum, the Institute for Infocomm Research (I2R), Microsoft Corporation, Motorola Inc., and Philips Electronics North America (Philips)) have been evaluated in detail for digital TV (DTV) scanning and spectrum-sensing capabilities, and field tests have been performed. In general, all the white-space devices have been capable of perfectly detecting (with $100 \%$ successful detection performance) clean DTV signals at input DTV powers larger than $-110 \mathrm{dBm}$, while some of them achieved $100 \%$ successful detection performance at input DTV powers lower than $-125 \mathrm{dBm}$. As such, the FCC report states that the burden of "proof of concept" has been met. Authorized spectrum-sensing devices in combination with geo-location and database access techniques can be used today, and devices relying on sensing alone may be addressed in the near future [74].

Several experimental studies specific to spectrum sensing have also been reported in the literature [75-77]. For instance, in [75], an energy-detector-based wireless test bed was developed to measure the required sensing time needed to achieve the desired probability of detection and false alarm for signals in a low-SNR region. The measurement results showed that a target probability of detection of $0.8\left(P_{d}=0.8\right)$ with the probability of false alarm set to $5 \%\left(P_{f a}=5 \%\right)$ could be achieved for signals greater than $-104 \mathrm{dBm}$ within a $170 \mathrm{~ms}$ sensing time.

\section{Location-Aware Systems}

The essential requirements for goal-driven and autonomous location-aware systems are accuracy, continuity, availability, and integrity $[5,78]$. In order for cognitive radio to support such systems, a conceptual model for a location-awareness engine was proposed in [5] and [43]. The main functionalities of a locationawareness engine are location sensing (or a cognitive positioning system, CPS), adaptation of location-aware systems, seamless positioning and interoperability, security and privacy, statistical learning and tracking, mobility management, location-aware applications, and a location-awareness core. The discussion in this section will focus on architectures, fundamental limits, challenges, mitigation approaches, and open issues for cognitive positioning systems and seamless positioning systems.

\subsection{Cognitive Positioning Systems}

A cognitive positioning system performs location-sensing functionality in a location-awareness engine. In other words, its main task is to provide the positioning accuracy specified by the cognitive engine. In order to meet various accuracy requirements, range-accuracy adaptation can be achieved by the adaptation of system parameters [2].

One way to provide range-accuracy adaptation in cognitive positioning systems involves the use of Cramer-Rao lower bounds
(CRLBs) for the optimization of signal parameters. As an illustrative example, consider a cognitive positioning system that performs range calculations based on time-of-arrival (TOA) estimation in a single-path additive white Gaussian noise (AWGN) channel environment. In this scenario, the Cramer-Rao lower bound on the variance of any unbiased range estimator $\hat{d}$ is given by [79]

$$
\sqrt{\operatorname{Var}\{\hat{d}\}} \geq \frac{c}{2 \sqrt{2} \pi \sqrt{S N R} \beta},
$$

where $S N R$ is the signal-to-noise ratio, $c$ is the speed of light, and $\beta$ is the effective signal bandwidth, defined as

$$
\beta^{2}=\frac{\int_{-\infty}^{\infty} f^{2}|S(f)|^{2} d f}{\int_{-\infty}^{\infty}|S(f)|^{2} d f}
$$

with $S(f)$ denoting the Fourier transform of the transmitted signal. From Equation (1), it can be observed that the transmitter can adapt the ranging accuracy by adjusting the SNR and/or the signal bandwidth based on feedback from the receiver. Note that the SNR can be adapted in a number of ways, such as by changing modulation type and the number of symbols that are transmitted for timeof-arrival estimation, i.e., by adjusting the ranging signal duration, and by changing the signal power [47].

Let's consider a numerical system design for the range-accuracy adaptation in a cognitive-radio system with the following system parameters. Assume that the cognitive-radio system has a capability to adapt the SNR by changing signal power between the values of $P_{t x, 1}$ and $P_{t x, 2}$, where $P_{t x, 1}$ and $P_{t x, 2}$ are the minimum and maximum transmitted power levels, respectively. In addition, the cognitive-radio system also has a capability to adapt effective bandwidth between the values of $\beta_{1}$ and $\beta_{2}$, where $\beta_{1}$ and $\beta_{2}$ are the minimum and maximum available effective bandwidths, respectively. The Cramer-Rao lower bound expression in Equation (1) for system parameters $P_{t x, 2}=4 P_{t x, 1}$ and $\beta_{2}=8 \beta_{1}$ is plotted in Figure 5. (It is assumed that $|S(f)|=1$ for the sake of simplicity.) According to the results, the cognitive radio can theoretically adapt the range accuracy between the bounds of $C R L B_{1}$ and $C R L B_{2}$. In other words, a range-accuracy adaptation between $58.88 \mathrm{~m}$ and $0.19 \mathrm{~m}$ can be achieved for the given system parameters. Another observation is that the theoretical operational region is quantified to be $24 \mathrm{~dB}$, which is simply the region between these two bounds. ( $6 \mathrm{~dB}$ is due to power gain $\left(P_{t x, 2} / P_{t x, 1}=4\right)$, and $18 \mathrm{~dB}$ is due to bandwidth gain $\left.\left(\beta_{2}^{2} / \beta_{1}^{2}=64\right)\right)$.

Note that practical systems cannot always perform very closely to the Cramer-Rao lower bounds. However, it is still reasonable to use these bounds as a metric for adaptation, by considering certain margins between the bounds and the system's performance. In addition, it is known that maximum-likelihood-(ML) and maximum-a-posteriori- (MAP) based location estimators can asymptotically achieve the Cramer-Rao lower bounds in line-ofsight (LOS) and non-line-of-sight (NLOS) environments, respectively [80]. Specifically, for high SNRs and/or large signal bandwidths, the positioning accuracy of a cognitive receiver can get very close to the theoretical limits. As an illustration of this issue, the performance of practical maximum-likelihood range-accuracy adaptation was evaluated for the previous system parameters, and is compared to the theoretical bounds in Figure 5. As can be seen from the figure, the performance of the practical maximum-likeli- 
hood range-accuracy adaptation depended on the SNR region [81, 82].

The SNR threshold phenomenon divides the region into two sub-regions. The regions before and after the SNR threshold $\left(S N R_{t h}\right)$ are called the nonlinear and linear regions, respectively. Note that practical maximum-likelihood range-accuracy adaptation asymptotically achieves the Cramer-Rao lower bound in Equation (1) in the linear region. In other words, the operational region for practical maximum-likelihood range-accuracy adaptation is mainly determined by the SNR thresholds, which are $S N R_{t h 1}=11 \mathrm{~dB}$ for $M L_{1}$ and $S N R_{t h 2}=17 \mathrm{~dB}$ for $M L_{2}$, in this case. In addition, cognitive-radio systems can practically perform rangeaccuracy adaptation between $8.32 \mathrm{~m}$ and $0.19 \mathrm{~m}$. Therefore, it is concluded that practical maximum-likelihood range-accuracy adaptation is desirable for operating in this linear region. (ZivZakai lower-bound- (ZZLB) based range-accuracy adaptation can perform very closely to practical systems in both nonlinear and linear regions $[83,84]$.) The performance of practical maximum-likelihood range-accuracy adaptation methods can be improved in terms of achieving the Cramer-Rao lower bounds asymptotically in several ways. One approach is to use the dispersed spectrum-utilization method, which is discussed later, in Section 6. Another approach is to use practically realizable lower bounds, such as the Ziv-Zakai lower bound [83], as an optimization criterion for rangeaccuracy adaptation methods.

The expression in Equation (1) illustrates the possibility of accuracy adaptation in cognitive positioning systems for singlepath additive-white-Gaussian-noise channels. However, practical cognitive positioning systems commonly operate in more-complicated propagation environments. In addition, they have dynamic operational characteristics driven by specific goals of the system. For example, a cognitive positioning system can utilize a number of dispersed frequency bands in the spectrum (Figure 6), and can perform range estimation based on signals received from the dispersed bands. Furthermore, a cognitive-radio channel can be quite dynamic; hence, a cognitive receiver might need to employ certain adaptive-positioning algorithms. Therefore, the dynamic operational characteristics of cognitive systems need special attention from the viewpoint of a cognitive positioning system.

\subsection{Seamless Positioning Systems}

It is well known that the performance of positioning systems can be significantly affected during the transition from one type of propagation environment to another one (e.g., the transition from an outdoor to an indoor environment) [45]. Therefore, seamless positioning is an approach that enables cognitive positioning systems to seamlessly operate in any environment. In general, seamless positioning is defined as a system that maintains the accuracy, integrity, continuity, and availability requirements of the cognitive engine, regardless of changes in propagation environment. However, the performance metric of interest will be range accuracy in this discussion. Consequently, the main objective of the seamless positioning system is to maintain the range accuracy at a predefined level in any propagation environment.

In this section, two main approaches for seamless positioning systems are discussed: Environmental-sensing-based methods, and waveform-based methods [5]. The main idea behind the first approach is to jointly estimate the range parameters (e.g., the path delay in the time-of-arrival estimation scenario) and the channel parameters, such as the path-loss exponent $(n)$ [85] and the frequency-dependence coefficient of the channel $(\kappa)$ [45]. In this approach, the effects of the channel environment on the range accuracy are inherently incorporated into the range estimation, since the channel parameter reflects the changes in the propagation environment. In [85], an RSS-based location-estimation method that jointly estimated the $\left[\begin{array}{ll}x & y\end{array}\right]$ coordinate information and $n$ was proposed. It was shown that the proposed algorithm kept the predefined position accuracy at a constant level, regardless of different $n$ values. Furthermore, seamless positioning systems based on the estimation of $\kappa$ for the channel propagation environment can be developed. In this line, Qiu et. al. extended the conventional complex channel model for wireless propagation by including the frequency-dependent feature of the signal path, which can provide physical insights into the channel environment. The channel's transfer function, including the frequency-dependent feature of signal paths, is defined as [86]

$$
H(w)=\sum_{l=1}^{L} \alpha_{l} e^{j \phi_{l}} e^{-j w \tau_{l}} w^{v_{l}},
$$

where $L$ is the number of paths, and $\alpha_{l}, \phi_{l}, \tau_{l}$, and $v_{l}$ are, respectively, the distance-dependent path coefficient, phase, delay, and frequency-dependent path coefficient of the lth path. In [86], singular-value decomposition along with the eigen-matrix pencil method was proposed to jointly estimate the $\alpha_{l}, \phi_{l}, \tau_{l}$, and $v_{l}$ parameters. Furthermore, according to the results reported from channel-measurement campaigns, each channel environment has different $\kappa$ values. For example, the channel parameters for the IEEE 802.15.4a channel measurements are tabulated in Table 1 [87]. In these results, it was assumed that all the paths had the same frequency-dependent distortion, i.e., $v_{l}=-2 \kappa$. This implies that $\kappa$ is one of the characteristic parameters for a channel environment. Hence, seamless positioning systems based on $\kappa$ estimation can be developed.

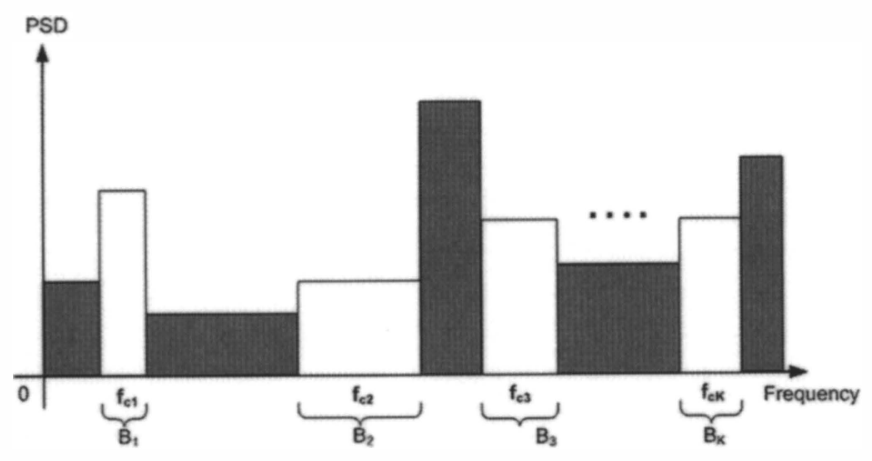

Figure 6. An illustration of dispersed spectrum utilization in cognitive-radio systems.

Table 1. The measured $\kappa$ values of some ultra-wideband (UWB) channels [87].

\begin{tabular}{|l|c|}
\hline Channel Models & $\kappa$ \\
\hline CM1 Residential Indoor LOS & 1.12 \\
\hline CM2 Residential Indoor NLOS & 1.53 \\
\hline CM3 Office Indoor LOS & 0.03 \\
\hline CM4 Office Indoor NLOS & 0.71 \\
\hline CM5 Outdoor LOS & 0.12 \\
\hline CM6 Outdoor NLOS & 0.13 \\
\hline CM7 Industrial LOS & -1.103 \\
\hline CM8 Industrial NLOS & -1.427 \\
\hline
\end{tabular}


Since estimation of a single channel parameter may not give sufficient information regarding the channel's environment, multiple parameters related to the channel's environment can be estimated for seamless positioning. This requires development of rapid and low-complexity signal-processing techniques.

The second approach for seamless positioning is the waveform-based method. This method selects the most-appropriate waveforms for given accuracy requirements and environment types [88]. This requires a cognitive-radio system equipped with multiple waveforms, such as global positioning system (GPS), Galileo, $3 \mathrm{G}$, ultra-wideband (UWB), wireless local-area-network (WLAN), and Bluetooth systems. The readers are referred to [88] for further details.

\section{Environmentally Aware Systems}

Environmental awareness is one of the most crucial functionalities of a cognitive-radio system, since the channel's environment is the bottleneck of wireless-communications systems. In order for cognitive-radio systems to interact and learn the surrounding environment, a conceptual model for an environmental-awareness engine (c.f. Figure 1) was proposed in [5]. In essence, the subsystem of the model corresponded to the main entities of an environment, which are topographical information, object recognition and tracking, propagation-channel characteristics, meteorological information, environmental sensing, environmentally aware applications, and an environmental-awareness engine core. The environmental-awareness engine core (which acts like the radio-environment "mapper" in [41]) collects all the environmental information from each aforementioned subsystem to form the complete radio-environment map at a given time.

In this section, the implementation options and challenges for environmental sensing and mapping using cognitive-radar functionalities of the environmental-awareness engine are discussed.

\subsection{Cognitive Radar}

In environmentally aware systems, cognitive radars can be used to provide information related to the objects in an environment, which can be considered to be an environmental sensing method. The main task of a cognitive radar is to provide object detection, identification, and tracking capabilities - based on intelligent signal-processing techniques - and feedback from the receiver to the transmitter $[39,89]$. Unlike conventional radars, a cognitive radar "learns" its environment, and preserves the information content of radar returns by applying Bayesian approaches [79].

A generic block diagram of a cognitive radar is illustrated in Figure 7. The transmitter illuminates the environment by sending a specific signal to the environment, and the signal reflections, called radar returns, are collected by the receiver. The radar returns contain information related to the environment, i.e., to the objects in the environment. In addition to the radar returns, the receiver can collect information-bearing signals that are present in the environment, such as temperature and rain intensity (i.e., meteorological information) [39]. By having current and forecasted meteorological information, such as rain intensity and temperature, a cognitive radio can accordingly adapt itself [5]. For instance, rain can have significant effects on the performance of broadband fixed wireless-

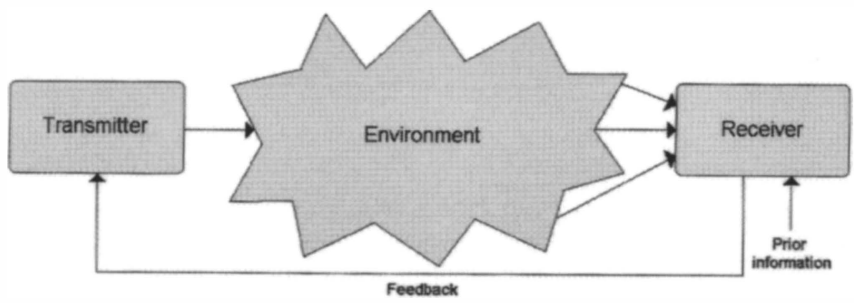

Figure 7. A block diagram of a cognitive-radar system.

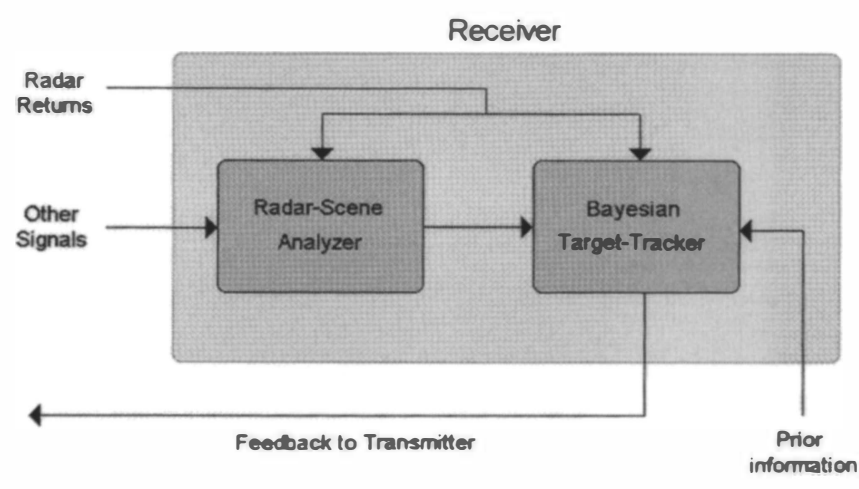

Figure 8. A block diagram of a cognitive-radar receiver.

access links (e.g., fixed WiMAX) [90], especially operating at higher carrier frequencies. One of the performance parameters that can be affected by rain is the carrier-to-interference ratio $(\mathrm{C} / \mathrm{I})$, and this performance metric depends on the rain intensity along the location of the desired signal path and the interferer signal paths. Some of the representative scenarios showing the rain effects on $\mathrm{C} / \mathrm{I}$ performance of broadband fixed wireless-access links are given as follows [90]: rain-induced $\mathrm{C} / \mathrm{I}$ degradation, rain-induced $\mathrm{C} / \mathrm{I}$ improvement, and no $\mathrm{C} / \mathrm{I}$ change. The details of these scenarios can be found in [90]. If a cognitive radio or network has a capability to acquire the rain intensity of local regions from a central meteorological server or the Internet, then $\mathrm{C} / \mathrm{I}$ adaptation can accordingly be performed [5]. Furthermore, according to an NSFsponsored study performed by the University of North Texas [91], the temperature and humidity affect the received signal strength (RSS). It was observed that higher temperature can lead to a slight decrease in received signal strength, and there is a 2 to $5 \mathrm{dBm}$ received signal strength loss as the temperature goes from $25^{\circ} \mathrm{C}$ to $45^{\circ} \mathrm{C}$. In addition, as humidity increases, the received signal strength becomes stronger. As a result, the cognitive radio can adapt the link according to the temperature and humidity in the operating environment. Note that he main task of the receiver is to use all the incoming signals and the prior information about the environment in order to perform target detection, identification, and tracking.

An important property of a cognitive-radar system is the presence of feedback from the receiver to the transmitter. Based on the feedback from the receiver, the transmitter can be adapted for improved performance. In other words, intelligent signal processing is facilitated by the presence of feedback [39]. Note that the feedback can be easily provided for monostatic radars, i.e., when the transmitter and the receiver are co-located. However, for bistatic radars, the design of feedback mechanisms can be challenging.

The main blocks of a cognitive-radar receiver are the radarscene analyzer and the Bayesian target tracker, as shown in Figure 8 . The radar-scene analyzer is the unit that extracts information 


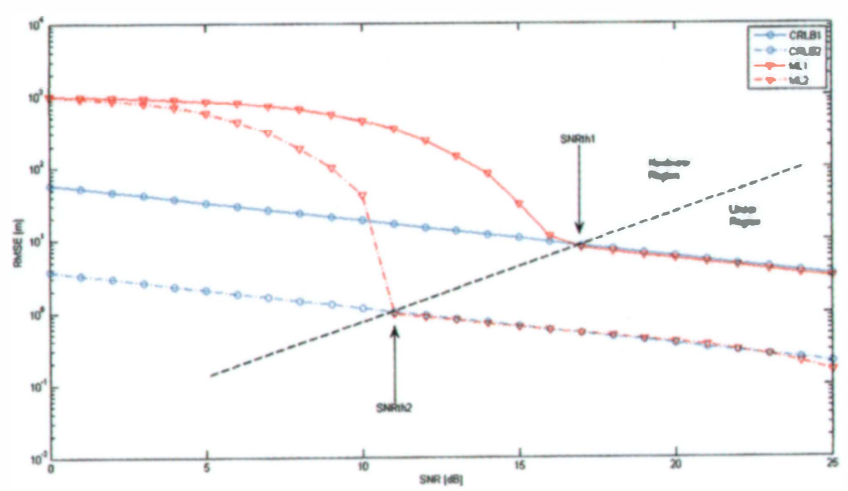

Figure 5. Operational regimes for range-accuracy adaptation.

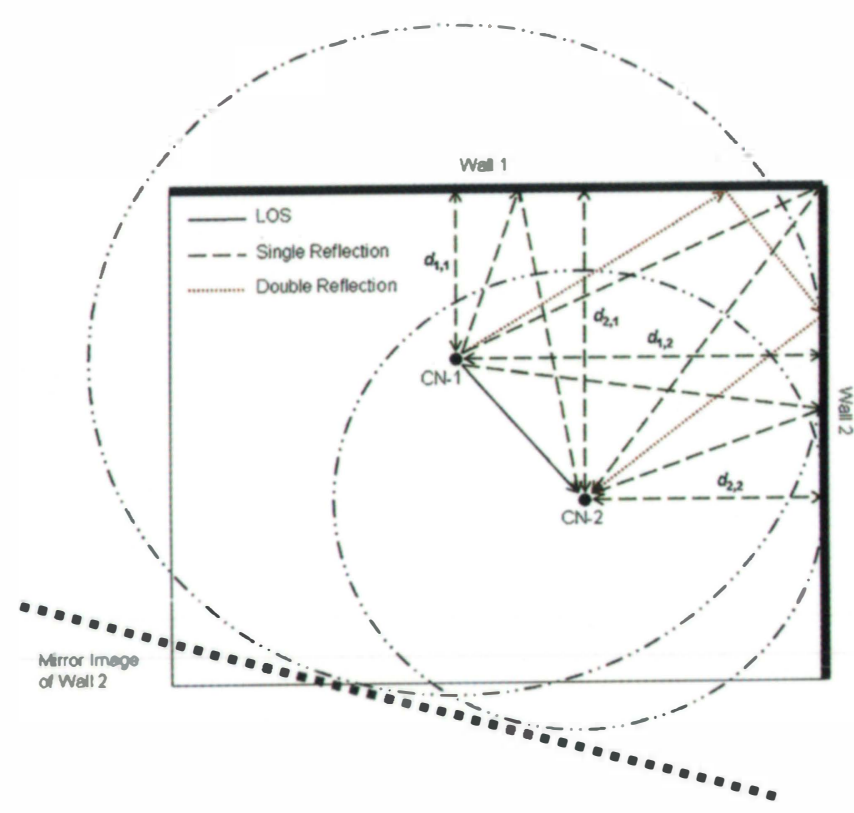

Figure 9. Two-dimensional indoor mapping without any infrastructure (modified from [54]). 
about the environment based on radar returns and other signals obtained from the environment [39]. The Bayesian-target-tracker unit uses the radar returns, the available prior information, and the statistical information provided by the radar-scene analyzer in order to employ Bayesian strategies [79, 92] for object detection and tracking. (The information provided to the Bayesian target tracker by the radar-scene analyzer is commonly in the form of parameters characterizing certain statistical distributions related to various radar returns [39].) In other words, the Bayesian-targettracker unit updates the prior information about the objects in the environment, based on information provided by the radar-scene analyzer and the observed radar returns. Hence, it basically calculates posterior distributions of certain events (e.g., an object to be present at a certain position at a given time) in the environment, based on all the available information.

\subsection{Environmental Mapping and Topographical Information Estimation}

UWB technology is capable of providing high-data-rate and robust communications and high-precision positioning, due its numerous attractive features. In addition to the aforementioned applications, UWB technology is traditionally used for radar applications [93]. Furthermore, UWB technology has been considered an integral part of new emerging technologies, such as cognitiveradio systems [52, 60, 94-99]. In other words, the connection between UWB and cognitive-radio systems is in the interest of many studies in the literature, and UWB is considered a part of cognitive-radio systems in those studies. For instance, UWB was considered as a solution for underlay spectrum access [96], control channel [95], and active interference cancellation [97] issues in cognitive-radio systems. In addition, numerous UWB-based cognitive-radio test beds, such as $[31,52,99]$ have been developed for demonstrating different applications. As a result, UWB can be considered to be another waveform type in the waveform library of cognitive-radio systems. Apart from the above applications of UWB positioning and communications in cognitive-radio systems, the UWB radar concept can be applied to cognitive-radio systems towards the realization of environmental awareness, which is considered in this study.

It is not an easy task to acquire complete knowledge about the surrounding environment using a single method or technology since the environment consists of different entities: topographical information, object recognition and tracking, channel-propagation characteristics, and meteorological information. Therefore, it is a more-efficient approach to use different methods and technologies to acquire the information about each entity of the environment. For instance, UWB radar technology can be used to detect, identify, and track the objects in an environment. Note that UWB can provide such capabilities for short ranges, and extending the range of UWB technology is an open research topic. On the other hand, the topographical information of an environment can be acquired by cognitive radio using digital elevation models (DEMs) and geographical-information-system (GIS) databases. The details of this approach can be found in [49].

One of the important characteristics of a cognitive-radio system is that it can learn/map its physical surroundings and topography $[5,41]$, even in the absence of any infrastructure. A cognitiveradio device can achieve this through listening to the returns of its own transmitted signals, as well as the signals transmitted by other cognitive-radio devices. Having an accurate map of the environment may have multiple uses. It can be used for situation/environment awareness purposes, where, for example, a fire- fighter can be guided to find his or her way out of a burning building. In fact, the European-Union-funded project EUROPCOM $[100,101]$ envisions a scenario where UWB radio is used in emergency situations (particularly within large buildings) where the locations of the personnel are monitored in a control vehicle. Since the building map may not be available a priori, or it may change due to damaged walls, etc., RF signals can be used to obtain an upto-date map of the building.

Having an accurate map of the environment also improves the position-estimation accuracy of the cognitive-radio device. This issue is commonly referred as the simultaneous localization and mapping (SLAM) problem in the literature (particularly, in the robotics-research community), where a mobile node jointly and incrementally builds a map of the environment while simultaneously estimating its own location [53, 54]. In [53], it was proven that the simultaneous localization and mapping problem can be solved in such a way that the mapping uncertainty and the position uncertainty can be improved up to a fundamental limit determined by the initial position uncertainty. If the sensor measurements for a cognitive-radio device $i$ at time $t$ are given by range measurements $d_{i}$ and angle measurements $\psi_{i}$, a vector $\mathbf{v}_{t}=\left\{d_{1}, \psi_{1}, d_{2}, \psi_{2}, \ldots, d_{M}, \psi_{M}\right\}$ can be defined for $M$ features of the environment. The joint state space of the cognitive device and the environment is then given by [53]

$$
\mathcal{S}=\left\{x, y, \theta, x_{1}, y_{1}, x_{2}, y_{2}, \ldots, x_{M}, y_{M}\right\},
$$

where the tuple $(x, y, \theta)$ defines the pose of the cognitive-radio device, and $\left(x_{i}, y_{i}\right)$ is the position of the $i$ th feature of the environment. The simultaneous localization and mapping problem is then defined as the estimation of following posterior distribution [54]

$$
p\left(\mathcal{S} \mid \mathbf{v}_{t}, \mathbf{u}_{t}, \mathbf{v}_{t-1}, \mathbf{u}_{t-1}, \ldots, \mathbf{v}_{0}, \mathbf{u}_{0}\right)
$$

where $\mathbf{u}_{t}=\left(\Delta d_{t}, \Delta \theta_{t}\right)$ is the relative transitional and rotational displacement of the cognitive radio device at time $t$.

In [55-57], UWB transceivers were used for simultaneous localization and mapping of a mobile node in simple two-wall and four-wall scenarios. The advantage of UWB for simultaneous localization and mapping is that due to their large bandwidths, UWB signals can resolve individual returns from different reflections with high timing accuracy. Therefore, the information embedded within distinct multipath components can be extracted, which is not possible with narrowband radios. Moreover, since a small number of dominant echoes corresponds to large flat surfaces such as walls, these dominant components can be captured by an appropriate thresholding method [55]. (Several other lesssignificant multipath components are typically scattered from smaller objects.)

Figure 9 illustrates a simple scenario in which two cognitiveradio nodes (CNs) can receive line-of-sight or reflected signals from each other (or reflections of their own signals) [55]. By processing different echoes, the mobiles can obtain the distances to each other as well as to the walls. In this scenario, $\mathrm{CN}-1$ receives three replicas of its own transmitted signal after reflections from the walls: one from wall-1, another from wall-2, and a third one from around the intersection of wall-1 and wall-2, which is a double reflection (not shown in Figure 9). While $\mathrm{CN}-1$ cannot distinguish these, it can obtain three distance measurements, $d_{1, j}$, $j \in\{1,2,3\}$. Similarly, $\mathrm{CN}-2$ receives three different echoes of its 
own transmitted signal to obtain distance measurements $d_{2, j}$, $j \in\{1,2,3\}$. Note that corresponding to each distance measurement, a position circle can be drawn around a $\mathrm{CN}$, and the tangents to this circle are possible locations of a wall. If the distance between $\mathrm{CN}-1$ and $\mathrm{CN}-2$ is known, the common tangents to the two position circles for $\mathrm{CN}-1$ and $\mathrm{CN}-2$ yield possible locations for a wall. For example, for measurements $d_{1,2}$ and $d_{2,2}$, there are two common tangents: one corresponds to wall-2, and the second one is the mirror image of wall-2 with respect to the line passing from $\mathrm{CN}-1$ and $\mathrm{CN}-2$. Since there are three measurements for each $\mathrm{CN}$, this corresponds to $3 \times 3 \times 2=18$ total hypotheses for the two walls, including the mirror images [54]. The ambiguity can be resolved by checking if any two wall combinations predict the double reflections with appropriate delays, and can yield the channel impulse response (CIR) from the other $\mathrm{CN}$. As apparent from the above discussion, the key parameter for finding the locations of the walls is the distance between two CNs. This parameter can be estimated by utilizing the channel impulse response between the two CNs, which has four echoes: the line-of-sight signal, two single reflections, and one double reflection. For further details and a discussion for a four-wall scenario, the reader is referred to [55].

\section{Dynamic Operation of Cognitive-Radio Systems}

In this section, the dynamic nature of cognitive-radio systems is discussed in the context of cognitive positioning systems. More specifically, the issues related to the dynamic nature of cognitive positioning systems are considered, and performance limits, challenges, mitigation algorithms, and adaptation techniques are discussed.

\subsection{Dynamic Spectrum Utilization}

Cognitive-radio systems facilitate opportunistic use of spectral resources. In other words, a cognitive-radio system can employ signals with dispersed frequency spectra, as shown in Figure 6. Therefore, the ranging accuracy of cognitive positioning systems depends also on the number of available frequency bands, and the bandwidth and center frequency of each of these bands.

In order to investigate the performance of cognitive positioning systems in dynamic spectrum-access scenarios, consider a cognitive-radio system that allocates $K$ different frequency bands, as in Figure 6 [47]. Assume a cognitive positioning receiver with $K$ different branches, each of which processes one of the $K$ frequency bands: i.e., each branch filters, amplifies, and down-converts the signal according to one of the center frequencies. (Another way to utilize dispersed spectra in cognitive positioning systems is to consider the received signal as an orthogonal frequency-division multiplexing (OFDM) signal, with zero coefficients at the sub-carriers corresponding to the unavailable bands [102-104]. Such an approach can require processing of very large bandwidths when the available spectrum is quite dispersed [47].). Then, the baseband representation of the received signal in the $i$ th branch can be expressed as

$$
r_{i}(t)=\alpha_{i} \mathrm{e}^{-j \omega_{i} t} s_{i}(t-\tau)+n_{i}(t)
$$

for $i=1, \ldots, K$, where $s_{i}(t)$ is the baseband representation of the transmitted signal corresponding to the $i$ th band, $\alpha_{i}=a_{i} \mathrm{e}^{j \phi_{i}}$ and $\omega_{i}$ respectively represent the channel coefficient and the carrierfrequency offset (CFO) for the signal in the $i$ th branch, $\tau$ is the time of arrival, and $n_{i}(t)$ is complex Gaussian noise with independent and white components, each having spectral density $\sigma_{i}^{2}$

In [47], the Cramer-Rao lower bounds were derived for the signal model in Equation (6) for the cases of known and unknown carrier-frequency offsets. As an example, for known carrier-frequency of fsets, the lower limit on the variance of unbiased timedelay estimators was expressed as follows:

$$
C R L B=\left[\sum_{i=1}^{K} \frac{\left|\alpha_{i}\right|^{2}}{\sigma_{i}^{2}}\left(\tilde{E}_{i}-\hat{E}_{i}^{2} / E_{i}\right)\right]^{-1}
$$

where

$$
E_{i}=\int_{0}^{T}\left|s_{i}(t-\tau)\right|^{2} d t
$$

is the signal energy, and

$$
\begin{aligned}
& \tilde{E}_{i}=\int_{0}^{T}\left|s_{i}^{\prime}(t-\tau)\right|^{2} \mathrm{~d} t, \\
& \hat{E}_{i}=\left|\int_{-\infty}^{\infty} s_{i}^{\prime}(t-\tau) s_{i}^{*}(t-\tau) \mathrm{d} t\right| .
\end{aligned}
$$

In other words, the Cramer-Rao lower bound depends on the SNRs of the available frequency bands, as well as on the properties of the ranging signal. In fact, it can be shown that for linearly modulated ranging signals with constant envelopes, the effects of unknown carrier-frequency offsets and channel coefficients can be mitigated for certain pulse shapes [47]. Therefore, range accuracy can be adapted in cognitive positioning systems by selecting the modulation type in addition to adjusting the number of ranging symbols, SNR levels, and/or the number of dispersed bands in the spectrum.

As an example, the Cramer-Rao lower bound in Equation (7) was plotted in Figure 10 as a function of the number of dispersed bands, where each band was assumed to have the same SNR and bandwidth [47]. Specifically, the received signal in each branch was modeled as a 16-PSK modulated sequence of 16 symbols. (For

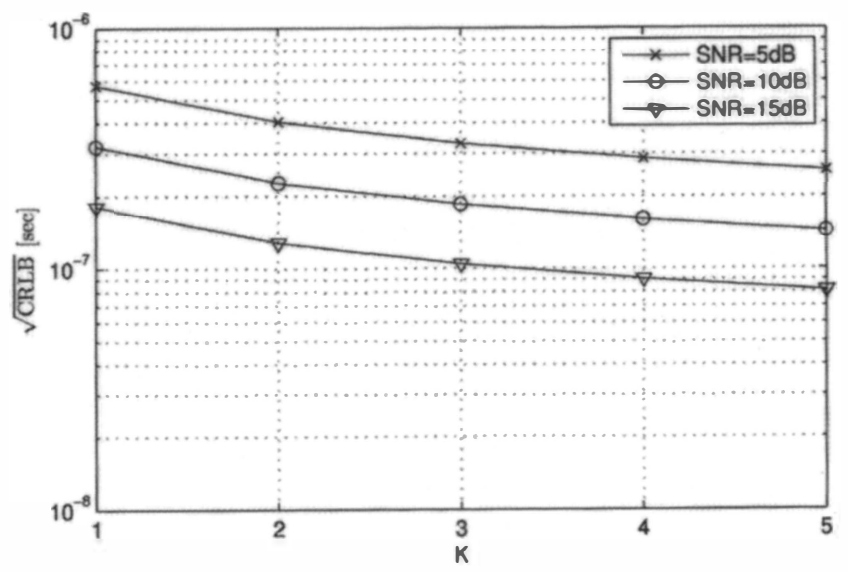

Figure 10. $\sqrt{C R L B}$ as a function of $K$ for 16PSK modulation. 
the pulse shape, a Gaussian doublet with a pulse width of $2.5 \mu \mathrm{s}$ was employed, and each bandwidth was considered to be $1 \mathrm{MHz}$ [47].). As could be observed from the figure, as more bands became available, better time-of-arrival-estimation accuracy could be achieved.

\subsection{Dynamic Transmission}

As discussed in the previous subsection, cognitive positioning systems that perform dynamic spectrum utilization provide a number of ways to perform adaptation of transmission parameters. For example, the modulation type, the SNR, the number of dispersed bands, and the duration of a ranging signal can be adapted to meet certain range-accuracy requirements.

Although in general positioning accuracy improves as the accuracy of range estimation increases, it also depends on other factors, such as the geometry/configuration and the number of nodes that are involved in position estimation. Since range-accuracy adaptation was studied in the previous subsection, positioning-accuracy adaptation based on transmission parameters and system configuration is investigated here.

In order to illustrate how parameter adaptation can be performed to meet a specific requirement on positioning accuracy, a cognitive-radio system that estimates position based on time-ofarrival measurements is considered. In a single-path additivewhite-Gaussian-noise channel with $N$ reference nodes, the CramerRao lower bound on the mean square error (MSE) of an unbiased estimator for the position of the target node can be expressed as [80]

$$
C R L B=\frac{c^{2} \sum_{i=1}^{N} S N R_{i}}{8 \pi^{2} \beta^{2} \sum_{i=1}^{N} \sum_{j=1}^{i-1} S N R_{i} S N R_{j} \sin ^{2}\left(\psi_{i}-\psi_{j}\right)},
$$

where $S N R_{i}$ represents the SNR of the signal related to the $i$ th reference node; $\beta$ is the effective bandwidth, which depends on the spectral contents of the signal used for time-of-arrival estimation [79]; and $\psi_{i}$ is the angle between the target node and the $i$ th reference node.

Note from Equation (11) that the Cramer-Rao lower bound depends on the system configuration/geometry, the number of reference nodes, the signal characteristics, and the SNRs [105]. In addition, $S N R_{i}$ is a function of the transmitted signal energy, the distance between a target node and the $i$ th reference node, the related propagation environment, and the spectral density of the noise. For a given system configuration and signal characteristics, the main method to maintain a Cramer-Rao lower bound that is lower than a specific requirement is to adjust the transmitted-signal energy employed in time-of-arrival estimation. The transmitted signal energy can be varied by changing the power of the signal [105], or by adjusting the signal duration for a fixed power level [106].

Although various techniques can be employed for SNR adaptation based on Equation (11), additional factors, such as nonline-of-sight (NLOS) conditions and multipath propagation, should be considered in practical scenarios. If the direct line of sight (LOS) between a reference node and the target node is blocked by an obstacle, the related time-of-arrival measurement can include a large bias, called non-line-of-sight error. This might degrade the positioning accuracy if that measurement is directly employed in a position estimator that is optimized for line-of-sight scenarios, such as in a least-squares (LS) estimator [66]. It can be shown that in the absence of any statistical information related to non-line-ofsight errors, the time-of-arrival measurements corrupted by these errors do not contribute to the positioning accuracy [80]. In other words, the Cramer-Rao lower bound in Equation (11) is still valid in these cases when $N$ is replaced by the number of line-of-sight reference nodes, and the calculations are performed only for lineof-sight measurements.

In the presence of statistical information related to non-lineof-sight errors, measurements from non-line-of-sight nodes should also be taken into account. In such cases, generalized Cramer-Rao lower bounds (G-CRLBs) can be employed in order to perform positioning-accuracy adaptation [80]. Note that the amount (accuracy) of statistical information related to non-line-of-sight error becomes an additional factor in determining the positioning accuracy of the system. Non-line-of-sight identification/mitigation issues in a cognitive positioning system will be discussed further in Section 6.4.

The previous discussions related to positioning-accuracy adaptation can also be extended to multipath environments. In multipath channels, the time-of-arrival estimation problem becomes the problem of estimating the delay of the first incoming signal path [107-109]. Since other paths can affect the accuracy of this estimation, the time of arrival, and hence the position-estimation accuracy, depends on the correlations between the first and the other received signal paths. Theoretical limits can be obtained in the absence and presence of prior statistical information about nonline-of-sight errors, similar to the previous discussions, and the corresponding Cramer-Rao lower bound and generalized CramerRao lower bound can be employed for positioning-accuracy adaptation [110]. (In addition to time-of-arrival-based positioning, one can also consider similar adaptation techniques for positioning systems that employ other position-related parameters, such as received signal strength and angle-of-arrival (AOA) [80, 111].)

\subsection{Dynamic Channel Environment}

In this subsection, a cognitive-radio channel is discussed from both information- and communication-theoretic perspectives. An information-theoretic view of cognitive-radio channels for dynamic spectrum access was introduced in [112, 113]. In these two studies, a two-transmitter and two-receiver channel was considered with asymmetric transmitter cooperation (or, cognition), where the secondary user transmitter had knowledge of the primary user's message prior to transmission. It was shown that the effects of cognition on the achievable rate of the channel depends on the SNR regime. For instance, cognition improves achievable rates over the interference channel in the medium-SNR region. On the other hand, the cognitive channel is interference limited in the high-SNR region. As a result, the cognitive and interference channels differ only from a transmitter cooperation point of view [112] In other words, the transmitters encode their messages independently in interference channels, whereas a secondary-user transmitter has a priori knowledge of the primary-user transmitter in the cognitive-radio channel. Such prior information in the cognitiveradio channel leads to achieving a degree of freedom close to two, which is equivalent to a $2 \times 2$ MIMO channel. 
The propagation-channel characterization is very crucial for wireless-system designs. The observed channel at the wireless receiver mainly depends on the transmission parameters and the surrounding environment. Hence, the first task for developing a wireless system (i.e., a transceiver) for a given environment and transmission parameters is to statistically model the propagation characteristics of the environment. A wireless propagation channel is generally described by two main sets of fading statistics: a) large-scale fading statistics, and b) small-scale fading statistics. The large-scale and small-scale fading statistics of a given channel's propagation environment are conventionally obtained by conducting channel-measurement campaigns. However, in cognitive-radio systems, transmission parameters are dynamic due to the dynamic available spectrum, and goal-driven and autonomousoperation features. Since the transmission parameters such as bandwidth, carrier frequency, and transmitted power are dynamic in cognitive-radio systems, the observed channel and corresponding statistics are also dynamic [5]. Furthermore, the changes in the environment, such as movement of the objects, result in an additional level of dynamism. Hence, it is a challenging task to realize and design wireless systems for such dynamic channel environments. As a result, there is a need to develop rapid and low-complexity channel-statistics acquisition methods.

Two potential approaches for channel-statistics acquisition in cognitive-radio systems are offline and online channel-statistics acquisition. In the first approach, the channel statistics are obtained from a cognitive base station for the given geographical area [50]. In this approach, it is assumed that cognitive-radio systems have their position information. A cognitive-radio system provides its transmission parameters and position information to the cognitive base station in order to obtain the channel-statistics information for its position. The cognitive base station then retrieves the channel statistics for the given position from a pre-built database by mapping the position to the corresponding channel-statistics information [50]. The main drawback of this approach is that it does not provide accurate small-scale fading statistics, as the objects in the given environment can be dynamic. However, it is possible to obtain large-scale fading statistics accurately by this approach. The second approach, which is the online method, is based on the idea of acquiring channel-statistics information between two cognitiveradio systems (i.e., a cognitive base station and a cognitive-radio node) in real time, which is a challenging task to achieve. As a result, development of rapid and low-complexity channel-statistics-acquisition methods is an active research area.

By having knowledge of channel statistics such as coherence time, coherence bandwidth, and delay spread in cognitive-radio systems, the performance can be improved by developing advanced algorithms. For instance, a coherence-time- $\left(T_{c}\right)$ based cognitive-radio adaptation method was proposed in [114]. In this method, the cognitive-radio system adapts every $T_{a}$ adaptation time units. If $T_{a} \leq T_{c}$, the cognitive-radio system adapts to smallscale fading statistics. If $T_{a}>T_{c}$, then the cognitive-radio system can only adapt to large-scale fading statistics. In addition, a modulation-adaptation method based on a coherence-time metric for a cognitive-radio system was proposed in [114]. In this method, the cognitive engine measures channel properties at every coherence time (i.e., "fade"), and then selects optimal transmission parameters such as modulation type. Note that in conventional radio systems, the optimal modulation rate is mainly selected based on the SNR metric, which provides inferior performance compared to the coherence-time metric used in cognitive-radio systems. In this context, it was shown that range accuracy in cognitive positioning systems can be adapted by using different modulation types [47].
Some further representative behaviors of cognitive-radio channels are provided in the context of cognitive positioning systems, as follows. One of the main parameters that affects the performance of a time-of-arrival-based cognitive positioning system is the utilized bandwidth. For instance, the transmission bandwidth can affect the path resolution of the channel, which can directly affect the positioning accuracy at the receiver side of the cognitive positioning system. Specifically, if a narrowband signal is used during the transmission, the path resolution decreases. Such effects were studied in [115] by deriving the Cramer-Rao lower bound for closely spaced paths. It was shown that the variance of a pathdelay estimate depends on the relative delays of all other path delays, not on their amplitudes. On the other hand, if the utilized transmission bandwidth is increased, the path resolution tends to increase. For instance, in the case of utilizing very large bandwidths (e.g., UWB signals [67]), the paths can be resolvable [116]. Since the utilized bandwidth in cognitive positioning systems can be dynamic, path resolutions become dynamic as well. As a result, dynamic path resolution is an impairment that can affect the performance of a cognitive positioning system. In summary, statistical modeling of cognitive-radio channel propagation considering a wide range of spectrum is crucial for the development of practical cognitive-radio systems, which is an active research area.

\subsection{Dynamic Reception}

Since transmission parameters and cognitive-radio channels are dynamic, receiver algorithms in cognitive positioning systems are also dynamic. In order to achieve a specific accuracy requirement, the receiver in a cognitive positioning system interacts with the transmitter through the location-awareness cycle for the optimization of transceiver parameters. Some examples of the adaptation mechanisms for location and range estimation in cognitiveradio systems are as follows.

\subsubsection{Adaptation Techniques for Range Estimation}

In range-based location estimation, a wireless device first has to estimate the distances, $\hat{d}_{i}(t)$, in Equation (12) from the received multipath signals. This is achieved by estimating the arrival time of the first multipath component. A common way to estimate this is by setting a threshold on the received signal samples. However, the threshold (as well as some other ranging parameters, such as the first path searching region) should be adaptive, and should change based on some characteristics of the transmitted/received signal. In the literature, some of the metrics that are used to adapt the threshold are noise variance, rms delay spread, signal energy, and kurtosis [107-109]. Moreover, the ranging algorithm in the location-awareness engine at the receiver should also adapt to the changes in the transmission bandwidth dictated by the spectrum-awareness engine and the arbitrary-waveform-generation block in Figure 1. While the impact of bandwidth on the accuracy of UWB ranging systems was analyzed in [117, 118], general adaptation mechanisms for accurate ranging at a cognitive-radio receiver that can cope with dynamic transmission bandwidth are still an open research area. (Note that the employed band groups may be dispersed over the spectrum.) 


\subsubsection{Adaptation Techniques for Location Estimation}

The location of a wireless node can be estimated in various ways from a set of distance (range) measurements from a number of reference nodes. For example, the non-linear least-squares estimate $\hat{\mathbf{x}}(t)$ for the location of a target node at time $t$ is given by [119]

$$
\hat{\mathbf{x}}(t)=\underset{\mathbf{x}(t)}{\arg \min _{i=1}}\left\{\sum_{i}^{N} w_{i}(t)\left(\hat{d}_{i}(t)-\left\|\mathbf{x}(t)-\mathbf{x}_{i}\right\|\right)^{2}\right\},
$$

where $\hat{d}_{i}(t)$ denotes the measured distance with the $i$ th reference node at time $t ; i=1, \ldots, N$, denotes the location of the $i$ th reference node; and the weights, $w_{i}(t)$, can be chosen to reflect the reliability of the signal received at the $i$ th reference node at time $t$.

Note that the measurements $\hat{d}_{i}(t)$ are dynamic and can change over time, due to factors such as channel variations, changes in transmission parameters, and mobility of the cognitiveradio node. For improved accuracy, the weights $w_{i}(t)$ in Equation (12) can be updated adaptively, based on the reliability of the measurements at a certain time instant. For example, they can be selected as the inverse of the variance of distance measurements i.e., $w_{i}(t)=1 / \sigma_{i}^{2}(t)$. (Note that for independent distance measurements corrupted by Gaussian noise, this actually yields the maximum likelihood (ML) estimator [111].) Then, in the presence of non-line-of-sight bias for certain reference nodes, the variance of distance measurements will be larger, and this will automatically be taken into account in Equation (12) by assigning them smaller weights. Alternatively, the non-line-of-sight reference nodes can be detected and discarded, which yields $w_{i}(t)=0$ for non-line-ofsight reference nodes. The weights $w_{i}(t)$ can also be obtained by utilizing the information in multipath components of a received signal (which is well-suited for UWB signals, due to large numbers of multipath components) [120].

As discussed previously, mitigation of non-line-of-sight errors is critical for achieving accuracy close to Cramer-Rao lower bounds. In Figure 11, a general classification of different non-lineof-sight mitigation approaches employed by a cognitive engine is illustrated. In each approach, some a priori non-line-of-sight knowledge is required. (Note that there are also other algorithms that do not require explicit non-line-of-sight information [67], such as robust estimators [121], residual-based techniques [122], and techniques that use non-line-of-sight measurements to obtain a feasible region for the location estimate [123]. The reader is referred to [124] for an in-depth study of different non-line-of-sight mitigation techniques.) Probably the simplest way of mitigating the nonline-of-sight effects in localization is to identify the non-line-ofsight reference nodes and discard them during location estimation [125] (e.g., $w_{i}(t)=0$ for the non-linear least-squares estimator if node $i$ is in non-line-of-sight). In [80], it was shown that under some assumptions (e.g., perfect knowledge of line-of-sight/nonline-of-sight reference nodes), such an approach is preferable over the approach that uses measurements from non-line-of-sight reference nodes as well for location estimation. A second strategy to obtain accurate position and environmental information is to generate a database of measurements at various known positions. The information contained in the database about the environment can be utilized in various ways. One way is to obtain non-line-of-sight error statistics based on the position-measurement pairs in the database. From the non-line-of-sight error statistics, various estimation techniques, such as maximum-likelihood and weighted least squares (WLS), can be employed to obtain position information for new measurements taken by the cognitive-radio system. Another way to utilize the database is to perform nonparametric estimation techniques, also called mapping techniques, to directly perform non-line-of-sight mitigation and position estimation [67]. Examples of mapping techniques include $k$-nearest-neighbor $(k$ $\mathrm{NN}$ ), support vector regression (SVR), and neural networks [126131]. The main idea behind the mapping techniques is to determine a regression scheme based on a set of training data, and to then estimate position of a given device according to that regression function [111]. Thirdly, it may be possible to obtain estimates of non-line-of-sight bias values and subtract them from distance measurements to obtain bias-free measurements. For example, in [132], non-line-of-sight bias values were estimated using an interior-point optimization technique. A weighted-least-squares technique was then used to estimate the location of the target node through unbiased measurements.

In order to obtain a closed-form solution from Equation (12), it is possible to use the techniques proposed in [133] and obtain a linear set of equations. For linearizing the system of equations, a reference node has to be selected, which is commonly done randomly in the literature [123]. Another adaptation mechanism in a cognitive positioning system is to adaptively select this reference node as the node that has the smallest distance measurement to the target node [134]. This was shown to improve the positioning accuracy. The index of the reference node at a particular time instant is given by

$$
\hat{i}(t)=\arg \min _{i}\left\{\hat{d}_{i}(t)\right\}, i=1,2, \ldots, N
$$

The covariance matrix of the observations (which also changes with time and needs to be updated periodically) may also be utilized for further improving the accuracy in a linear-least-squares estimator [134].

A cognitive-radio receiver is also envisioned to mitigate the impacts of interference sources. In the presence of multiple-access interference (MAI), the range accuracy may degrade considerably. In such scenarios, it was shown in [135] that the ranging accuracy of a UWB receiver can be improved by employing nonlinear filters (e.g., a median filter or a minimum filter). Such filters are used in order to mitigate the effects of the outliers in the received signal

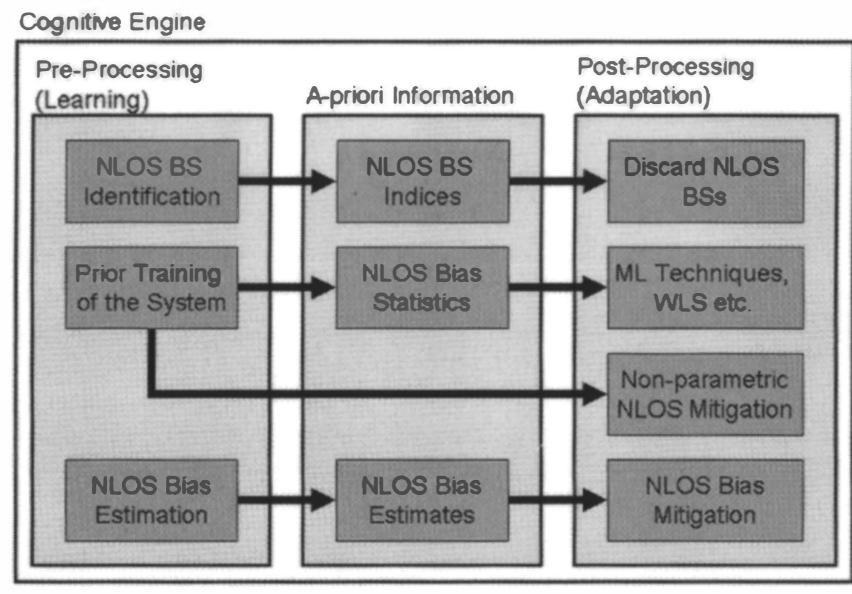

Figure 11. The classification of the main NLOS mitigation techniques that can be employed in a cognitive engine. 
Table 2. A list of representative references in the prior art related to spectrum, location, and environmentally aware systems.

\begin{tabular}{|l|l|l|}
\hline Category & \multicolumn{1}{|c|}{ Subcategory } & \multicolumn{1}{c|}{ References } \\
\hline \multirow{4}{*}{$\begin{array}{l}\text { Spectrum Aware } \\
\text { Systems }\end{array}$} & Single-Antenna Spectrum Sensing & $15-25$ \\
\cline { 2 - 3 } & Collaborative Spectrum Sensing & $26-33$ \\
\cline { 2 - 3 } & Multi-Antenna Spectrum Sensing & 34,35 \\
\hline \multirow{4}{*}{$\begin{array}{l}\text { Location Aware } \\
\text { Systems }\end{array}$} & General References & $5,43,60,81$ \\
\cline { 2 - 3 } & Cognitive Positioning Systems & $2,44,45$ \\
\cline { 2 - 3 } & Dynamic Spectrum Utilization & $7,8,10,47,137$ \\
\cline { 2 - 3 } & Dynamic Transmission & $80,105,106$ \\
\cline { 2 - 3 } & Dynamic Channel Environment & $5,50,138$ \\
\cline { 2 - 3 } & Adaptation Techniques for Location Estimation & $111,119,120,126,132,134$ \\
\cline { 2 - 3 } & Adaptation Techniques for Range Estimation & $107-109,117-136$ \\
\cline { 2 - 3 } & Seamless Positioning Systems & $5,45,85,86,88$ \\
\hline \multirow{2}{*}{$\begin{array}{l}\text { Environmentally } \\
\text { Aware Systems }\end{array}$} & General References & 5,50 \\
\cline { 2 - 3 } & Cognitive Radar & 39,89 \\
\cline { 2 - 3 } & Environment Mapping and SLAM & $41,53-57,101,139$ \\
\hline
\end{tabular}

samples before de-spreading the signal. A similar approach was extended to mitigate the effects of narrowband interference on the ranging accuracy in [136]. However, in the absence of any interference (or, at very high signal-to-interference ratios), the SNR of the received signal is degraded after processing it with a nonlinear filter. For the best accuracy, the cognitive engine may first detect the presence of an interferer. If an interferer is present, the cognitive engine can employ a nonlinear filter to mitigate the effects of the interference prior to range estimation. Otherwise, range is estimated without employing any outlier-detection techniques.

\section{Concluding Remarks}

A cognitive-radio system with spectrum, location, and environmental awareness features provides a promising mechanism for obtaining accurate spectrum, location, and environmental information. This paper has investigated the main functionalities of such systems. First, individual and collaborative spectrum sensing, using single and multiple antennas, was considered for spectrumaware systems. Cognitive positioning systems and seamless positioning systems were then studied in terms of location awareness. In addition, environment-aware systems were investigated, and cognitive-radar and environmental-mapping functionalities were studied. Furthermore, the dynamic operation of cognitive-radio systems in the context of cognitive positioning systems was presented. Finally, the main references for the prior art on spectrum, location, and environmentally aware systems have been summarized in Table 2. It is observed that accurate characterization of operational environments can be achieved by development of practical cognitive-radio systems with spectrum, location, and environmental awareness features. The analysis provided for the range-accuracy adaptation method can be extended to more-realistic scenarios, such as those with multipath and frequency-selective channels.

\section{Acknowledgments}

This work was supported in part by Qatar National Research Fund (QNRF) and Qatar Telecom (Qtel). S. Gezici wishes to acknowledge the activity of the Network of Excellence in Wireless COMmunications NEWCOM++ of the European Commission (contract n. 216715) that motivated this work.

\section{References}

1. J. Mitola and G. Q. Maguire, "Cognitive radio: Making Software Radios More Personal," IEEE Personal Communications Magazine, 6, 4, August 1999, pp. 13-18.

2. H. Celebi and H. Arslan, "Cognitive Positioning Systems," IEEE Transactions on Wireless Communications, 6, 12, December 2007, pp. 4475-4483.

3. "IEEE Standards Coordinating Committee 41," 2007; available at: http://www.ieep1900.org/.

4. J. O. Neel, Analysis and Design of Cognitive Radio Networks and Distributed Radio Resource Management Algorithms, PhD dissertation, Virginia Polytechnic Institute and State University, Blacksburg, VA, September 2006.

5. H. Celebi and H. Arslan, "Enabling Location and Environment Awareness in Cognitive Radios," Elsevier Computer Communications, 31, 6, April 2008, pp. 1114-1125.

6. J. Riihijarvi and P. Mahonen, "Exploiting Spatial Statistics of Primary and Secondary Users Towards Improved Cognitive Radio Networks," International Conference on Cognitive Radio Oriented Wireless Networks and Communications (CrownCom), Orlando, FL, USA, May 2008, pp. 1-7.

7. T. Yucek and H. Arslan, "A Survey of Spectrum Sensing Algorithms for Cognitive Radio Applications," IEEE Communications Surveys and Tutorials, 11, 1, March 2009, pp. 116-130.

8. I. F. Akyildiz, W. Y. Lee, M. C. Vuran, and S. Mohanty, "A Survey on Spectrum Management in Cognitive Radio Networks Cognitive Radio Communications and Networks," IEEE Communications Magazine, 46, 4, April 2008, pp. 40-48.

9. S. Haykin, "Cognitive Radio: Brain-Empowered Wireless Communications," IEEE Journal on Selected Areas in Communications, 23, 2, February 2005, pp. 201-220.

10. Q. Zhao and B. M. Sadler, "A Survey of Dynamic Spectrum Access," IEEE Signal Processing Magazine, 24, 3, May 2007, pp. 79-89. 
11. I. F. Akyildiz, W. Y. Lee, M. C. Vuran, and S. Mohanty, "Next Generation/Dynamic Spectrum Access/Cognitive Radio Wireless Networks: A Survey," Elsevier Computer Networks, 50, 13, September 2006, pp. 2127-2159.

12. Q. Zhao and A. Swami, "A Survey of Dynamic Spectrum Access: Signal Processing and Networking Perspectives," IEEE International Conference on Acoustics, Speech and Signal Processing, Honolulu, HI, 4, April 2007, pp. 1349-1352.

13. T. Ikuma and M. N. Pour, "A Comparison of Three Classes of Spectrum Sensing Techniques," Proceedings of the IEEE Global Telecommunications Conference, New Orleans, LA, November 2008, pp. 1-5.

14. T.-W. Wu, Y.-E. Lin, and H.-Y. Hsieh, "Modeling and Comparison of Primary User Detection Techniques in Cognitive Radio Networks," Proceedings of the IEEE Global Telecommunications Conference, New Orleans, LA, November 2008, pp. 1-5.

15. Z. Quan, S. Cui, A. H. Sayed, and H. V. Poor, "Wideband Spectrum Sensing in Cognitive Radio Networks," Proceedings of the IEEE International Conference on Communications, Beijing, China, May 2008, pp. 901-906.

16. W.-Y. Lee and I. F. Akyildiz, "Optimal Spectrum Sensing Framework for Cognitive Radio Networks," IEEE Transactions on Wireless Communications, 7, 10, October 2008, pp. 3845-3857.

17. C. Cordeiro, M. Ghosh, D. Cavalcanti, and K. Challapali, "Spectrum Sensing for Dynamic Spectrum Access of TV Bands," Proceedings of the IEEE International Conference on Cognitive Radio Oriented Wireless Networks and Communications, Orlando, FL, August 2007, pp. 225-233.

18. H.-S. Chen, W. Gao, and D. G. Daut, "Spectrum Sensing Using Cyclostationary Properties and Application to IEEE 802.22 WRAN," Proceedings of the IEEE Global Telecommunications Conference, Washington, DC, November 2007, pp. 3133-3138.

19. R. Tandra, A. Sahai, and S. M. Mishra, "What is a Spectrum Hole and What Does it Take to Recognize One?" Proceedings of the IEEE, 97, 5, May 2009, pp. 824-848.

20. S.-Y. Tu, K.-C. Chen, and R. Prasad, "Spectrum Sensing of OFDMA Systems for Cognitive Radios," Proceedings of the IEEE International Symposium on Personal, Indoor, Mobile Communications, Athens, Greece, September 2007, pp. 1-5.

21. C. Cordeiro, M. Ghosh, D. Cavalcanti, and K. Challapali, "Spectrum Sensing for Dynamic Spectrum Access of TV Bands," Proceedings of the IEEE International Conference on Cognitive Radio Oriented Wireless Networks and Communications, Orlando, FL, August 2007, pp. 225-233.

22. N. Khambekar, L. Dong, and V. Chaudhary, "Utilizing OFDM Guard Interval for Spectrum Sensing," Proceedings of the IEEE Wireless Communications and Networking Conference, Hong Kong, March 2007, pp. 38-42.

23. D. Chen, J. Li, and J. Ma, "In-Band Sensing Without Quiet Period in Cognitive Radio," Proceedings of the IEEE Wireless Communications and Networking Conference, Las Vegas, NV, April 2008, pp. 723-728.

24. L. Lv, Z. Wu, M. Jie, S. Y. Chang, J. Zhang, L. Qian, and J. Wen, "Orthogonal Interference Detection," November 2006, doc. No: IEEE 802.22-05/0262r0.
25. S. Xu, Z. Zhao, and J. Shang, "Spectrum Sensing Based on Cyclostationarity," Proceedings of the IEEE Workshop on Power Electronics and Intelligent Transportation System, Guangzhou, China, August 2008, pp. 171-174.

26. Z. Quan, S. Cui, and A. H. Sayed, "Optimal Linear Cooperation for Spectrum Sensing in Cognitive Radio Networks," IEEE Journal on Selected Topics in Signal Processing, 2, 1, February 2008, pp. 28-40.

27. C. Han Lee and W. Wolf, "Multiple Access-Inspired Cooperative Spectrum Sensing for Cognitive Radio," Proceedings of the IEEE Military Communications Conference, San Diego, CA, October 2007, pp. 1-6.

28. J. Ma and Y. Li, "Soft Combination and Detection for Cooperative Spectrum Sensing in Cognitive Radio Networks," Proceedings of the IEEE Global Telecommunications Conference, Washington, DC, November 2007, pp. 3139-3143.

29. Y. Wang, C. Feng, Z. Zeng, and C. Guo, "A Robust and Energy Efficient Cooperative Spectrum Sensing Scheme in Cognitive Radio Networks," Proceedings of the IEEE International Conference on Advanced Communication Technology, vol. 1, Phoenix Park, Korea, February 2009, pp. 640-645.

30. Z. Quan, S. Cui, and A. H. Sayed, "An Optimal Strategy for Cooperative Spectrum Sensing in Cognitive Radio Networks," Proceedings of the IEEE Global Telecommunications Conference, Washington, DC, November 2007, pp. 2947-2951.

31. W. Zhang, R. K. Mallik, and K. B. Letaief, "Cooperative Spectrum Sensing Optimization in Cognitive Radio Networks," Proceedings of the IEEE International Conference Communications, Beijing, China, May 2008, pp. 3411-3415.

32. C. Sun, W. Zhang, and K. Ben, "Cluster-Based Cooperative Spectrum Sensing in Cognitive Radio Systems," Proceedings of the IEEE International Conference on Communications, Glasgow, Scotland, June 2007, pp. 2511-2515.

33. W. Wang, L. Zhang, W. Zou, and Z. Zhou, "On the Distributed Cooperative Spectrum Sensing for Cognitive Radio," Proceedings of the IEEE International Symposium on Communications and Information Technology, Sydney, Australia, October 2007, pp. 1496-1501.

34. X. Chen, W. Xu, Z. He, and X. Tao, "Spectral CorrelationBased Multi-Antenna Spectrum Sensing Technique," Proceedings of the IEEE Wireless Communications and Networking Conference, Las Vegas, NV, April 2008, pp. 735-740.

35. J.-H. Lee, J.-H. Baek, and S.-H. Hwang, "Collaborative Spectrum Sensing Using Energy Detector in Multiple Antenna System," Proceedings of the IEEE International Conference on Advanced Communication Technology, 1, Phoenix Park, Korea, February 2008, pp. 427-430.

36. J. Lunden, V. Koivunen, A. Huttunen, and H. V. Poor, "Censoring for Collaborative Spectrum Sensing in Cognitive Radios," Proceedings of the IEEE Asilomar Conference on Signals, Systems and Computers, Pacific Grove, CA, November 2007, pp. 772-776.

37. V. Kuppusamy and R. Mahapatra, "Primary User Detection in OFDM Based MIMO Cognitive Radio," International Conference Cognitive Radio Oriented Wireless Networks and Communications, Singapore, May 2008, pp. 1-5. 
38. T. Zhang, G. Yu, and C. Sun, "Performance of Cyclostationary Features Based Spectrum Sensing Method in a Multiple Antenna Cognitive Radio System," Proceedings of the IEEE Wireless Communications and Networking Conference, Budapest, Hungary, April 2007, pp. 1-5.

39. S. Haykin, "Cognitive Radar: A Way of the Future," IEEE Signal Processing Magazine, 23, 1, January 2006, pp. 30-40.

40. S. Haykin, "Cognitive Radar Networks," Fourth IEEE Workshop on Sensor Array and Multichannel Processing, Boston, MA, July 2006, pp. 1-24.

41. Y. Zhao, H. H. Reed, S. Mao, and K. K. Bae, "Overhead Analysis for Radio Environment Mapenabled Cognitive Radio Networks," Proceedings of the IEEE Workshop on Networking Technologies for Software Defined Radio Networks, Reston, VA, September 2006, pp. 18-25.

42. N. Goodman, P. Venkata, and M. Neifeld, "Adaptive Waveform Design and Sequential Hypothesis Testing for Target Recognition with Active Sensors," IEEE Journal on Selected Topics in Signal Processing, 1, 1, June 2007, pp. 105-113.

43. H. Celebi and H. Arslan, "Utilization of Location Information in Cognitive Wireless Networks," IEEE Wireless Communications Magazine, 14, 4, August 2007, pp. 6-13.

44. H. Celebi and H. Arslan, "Adaptive Positioning Systems for Cognitive Radios," Proceedings of the IEEE Symposium on New Frontiers in Dynamic Spectrum Access Networks, Dublin, Ireland, April 2007, pp. 78-84.

45. H. Celebi and H. Arslan, "Ranging Accuracy in Dynamic Spectrum Access Networks," IEEE Communications Letters, 11, 5, May 2007, pp. 405-407.

46. D. Dardari, Y. Karisan, S. Gezici, A. A. D'Amico, and U. Mengali, "Performance Limits on Ranging with Cognitive Radio," IEEE International Conference on Communications Workshops, Dresden, Germany, June 2009, pp. 1-5.

47. S. Gezici, H. Celebi, H. V. Poor, and H. Arslan, "Fundamental Limits on Time Delay Estimation in Dispersed Spectrum Cognitive Radio Systems," IEEE Transactions on Wireless Communications, 8, 1, January 2009, pp. 78-83.

48. F. Kocak, H. Celebi, S. Gezici, K. A. Qaraqe, H. Arslan, and H. V. Poor, "Time-Delay Estimation in Dispersed Spectrum Cognitive Radio Systems," EURASIP Journal on Advances in Signal Processing, 2010, article ID 675959, 10 pages.

49. H. Celebi, K. A. Qaraqe, and H. Arslan, "Performance Comparison of Time Delay Estimation for Whole and Dispersed Spectrum Utilization Methods in Cognitive Radio Networks," International Conference on Cognitive Radio Oriented Wireless Networks and Communications, Hanover, Germany, June 2009, pp. 1-6.

50. S. Yarkan and H. Arslan, "Exploiting Location Awareness Towards Improved Wireless System Design in Cognitive Radio," IEEE Communications Magazine, 46, 1, January 2008, pp. 128136.

51. S. Haykin, “Cognitive Dynamic Systems," IEEE International Conference on Acoustics, Speech and Signal Processing, Honolulu, Hawaii, vol. 4, April 2007, pp. 1369-1370.
52. R. Qiu, N. Guo, H. Li, Z. Wu, V. Chakravarthy, Y. Song, Z. Hu, P. Zhang, and Z. Chen, "A Unified Framework for Cognitive Radio, Cognitive Radar, and Electronic Warfare: Tutorial, Theory, and Multi-GHz Wideband Testbed,"Sensors, 9, 2009.

53. M. W. M. G. Dissanayake, P. Newman, S. Clark, H. F. D. Whyte, and M. Csorba, "A Solution to the Simultaneous Localization and Map Building (SLAM) Problem," IEEE Transactions on Robotics and Automation, 17, 3, June 2001, pp. 229-241.

54. R. Sim and N. Roy, "Global A-Optimal Robot Exploration in SLAM," Proceedings of the IEEE International Conference on Robotics and Automation, Barcelona, Spain, April 2005, pp. 661666.

55. W. Guo, N. P. Filer, and S. K. Barton, "2D Indoor Mapping and Positioning Using an Impulse Radio Network," International Conference on Ultra-Wideband, Zurich, Switzerland, September 2005, pp. 296-301.

56. W. Guo, N. P. Filer, and R. Zetik, "Indoor Mapping and Positioning Using Impulse Radios," Proceedings of the IEEE Position, Location, and Navigation Symposium, San Diego, CA, April 2006, pp. 153-163.

57. W. Guo and N. P. Filer, "2.5D Indoor Mapping and LocationSensing Using an Impulse Radio Network," Proceedings of the IET Seminar on Ultra Wideband Systems, Technologies, and Applications, London, UK, April 2006, pp. 211-215.

58. T. Newman, B. Barker, A. Wyglinski, A. Agah, J. Evans, and G. Minden, "Cognitive Engine Implementation for Wireless Multicarrier Transceivers," Wireless Communications and Mobile Computing, 7, 9, May 2007, pp. 1129-1142.

59. R. Thomas, D. Friend, L. Dasilva, and A. Mackenzie, "Cognitive Networks: Adaptation and Learning to Achieve End-to-End Performance Objectives," IEEE Communications Magazine, 44, 12 , January 2006, pp. 51-57.

60. H. Arslan, Cognitive Radio, Software Defined Radio, and Adaptive Wireless Systems, New York, Springer, September 2007.

61. S. Oh, J. Aberle, S. Anantharaman, K. Arai, H. Chong, and S. Koay, "Electronically Tunable Antenna Pair and Novel RF FrontEnd Architecture for Software-Defined Radios," EURASIP Journal on Applied Signal Processing, 2005, 16, 2005, pp. 2701-2707.

62. W. Zhu, B. Daneshrad, J. Bhatia, J. Chen, H. Kim, K. Mohammed, O. Nasr, S. Sasi, A. Shah, and M. Tsai, "A Real Time MIMO OFDM Testbed for Cognitive Radio \& Networking Research," Proceedings of the 1st International Workshop on Wireless Network Testbeds, Experimental Evaluation \& Characterization, New York, ACM, 2006, pp. 115-116.

63. C. Rieser, Biologically Inspired Cognitive Radio Engine Model Utilizing Distributed Genetic Algorithms for Secure and Robust Wireless Communications and Networking, PhD dissertation, Virginia Polytechnic Institute and State University, Blacksburg, VA, August 2004.

64. C. Rieser, T. Rondeau, C. Bostian, and T. Gallagher, "Cognitive Radio Testbed: Further Details and Testing of a Distributed Genetic Algorithm Based Cognitive Engine for Programmable Radios," IEEE Military Communications Conference, 3, 2004. 
65. Z. Quan, S. Cui, H. Poor, and A. Sayed, "Collaborative Wideband Sensing for Cognitive Radios," IEEE Signal Processing Magazine, 25, 6, November 2008, pp. 60-73.

66. J. J. Caffery, Wireless Location in CDMA Cellular Radio Systems, Boston, Kluwer Academic Publishers, 2000.

67. Z. Sahinoglu, S. Gezici, and I. Guvenc, Ultra-Wideband Positioning Systems: Theoretical Limits, Ranging Algorithms, and Protocols, New York, Cambridge University Press, 2008.

68. M. A. Richards, Fundamentals of Radar Signal Processing, New York, McGraw-Hill, 2005.

69. N. Levanon and E. Mozeson, Radar Signals, New York, Wiley-IEEE Press, 2004.

70. B. Allen, M. Dohler, E. E. Okon, W. Q. Malik, A. K. Brown, and D. J. Edwards, Ultra-Wideband Antennas and Propagation for Communications, Radar, and Imaging, West Sussex, England, John Wiley \& Sons, Ltd., 2007.

71. A. Goldsmith, Wireless Communications, Cambridge, Cambridge University Press, 2005.

72. T. Peng, W. Wang, Q. Lu, and W. Wang, "Subcarrier Allocation Based on Water-Filling Level in OFDMA-Based Cognitive Radio Networks," Proceedings of the IEEE International Conference on Wireless Communications Networking and Mobile Computing, Shanghai, China, September 2007, pp. 196-199.

73. FCC, "Second Report and Order and Memorandum Opinion and Order (FCC 08-260)," November 2008, in the Matter of Unlicensed Operation in TV Broadcast Bands and Additional Spectrum for Unlicensed Devices Below $900 \mathrm{MHz}$ and in the $3 \mathrm{GHz}$ Band; available: http://hraunfoss.fcc.gov/edocs public/attachmatch/FCC08-260A1.pdf.

74. S. K. Jones, T. W. Phillips, H. L. V. Tuyl, and R. D. Weller, "Evaluation of the Performance of Prototype TV-Band White Space Devices Phase-II (FCC/OET 08-TR-1005)," October 2008.: Available: http://hraunfoss.fcc.gov/edocspublic/attachmatch/DA08-2243A3.pdf.

75. D. Cabric, A. Tkachenko, and R. Brodersen, "Experimental Study of Spectrum Sensing Based on Energy Detection and Network Cooperation," Proceedings of the First International Workshop on Technology and Policy for Accessing Spectrum, New York, ACM, 2006.

76. D. Cabric, A. Tkachenko, and R. Brodersen, "Spectrum Sensing Measurements of Pilot, Energy, and Collaborative Detection," Military Communications Conference, Washington, DC, October 2006, pp. 1-7.

77. M. Wellens, J. Riihijarvi, M. Gordziel, and P. Mahonen, "Evaluation of Cooperative Spectrum Sensing Based on Large Scale Measurements," Proceedings of the of IEEE Symposium on New Frontiers in Dynamic Spectrum Access Networks, Chicago, IL, USA, October 2008.

78. S. Feng and W. Y. Ochieng, "User Level Autonomous Integrity Monitoring for Seamless Positioning in All Conditions and Environments," Proceedings of The European Navigation Conference, Manchester, UK, May 2006.
79. H. V. Poor, An Introduction to Signal Detection and Estimation, New York, Springer-Verlag, 1994.

80. Y. Qi, H. Kobayashi, and H. Suda, "Analysis of Wireless Geolocation in a Non-Line-Of-Sight Environment," IEEE Transactions on Wireless Communications, 5, 3, March 2006, pp. 672 681.

81. H. Celebi, Location Awareness in Cognitive Radio Networks, $\mathrm{PhD}$ dissertation, University of South Florida, FL, August 2008.

82. A. Zeira and P. Schultheiss, "Realizable Lower Bounds for Time Delay Estimation II: Threshold Phenomena," IEEE Transactions on Signal Processing, 42, 5,'1994, pp. 1001-1007.

83. K. L. Bell, Y. Steinberg, Y. Ephraim, and H. L. V. Trees, "Extended Ziv-Zakai Lower Bound For Vector Parameter Estimation," IEEE Transactions on Information Theory, 43, 2, February 1997, pp. 624-637.

84. A. Weiss and E. Weinstein, "Fundamental Limitations in Passive Time Delay Estimation - Part I: Narrow-Band Systems," IEEE Transactions on Acoustics, Speech, and Signal Processing, 31, 2, 1983, pp. 472-486.

85. X. Li, "RSS-Based Location Estimation with Unknown Pathloss Model," IEEE Transactions on Wireless Communications, 5, 12, December 2006, pp. 3626-3633.

86. R. C. Qiu and I-T. Lu, "Multipath Resolving with Frequency Dependence for Wide-band Wireless Channel Modeling," IEEE Transactions on Vehicular Technology, 48, 1, January 1999, pp. 273-285.

87. A. F. Molisch, K. Balakrishnan, D. Cassioli, C. C. Chong, S. Emami, A. Fort, J. Karedal, J. Kunisch, H. Schantz, U. Schuster, and K. Siwiak, "IEEE 802.15.4a Channel Model - Final Report," 2005, Tech. Rep. Doc: IEEE 802.15-04-0662-02-004a; available: http://www.ieee802.org/15/pub/TG4a.html.

88. T. Moore, C. Hill, C. Hide, D. Walsh, J. Cooper, R. Ioannides, W. Ochieng, S. Feng, P. Cross, and L. Lau, "Seamless Positioning in All Conditions and Environments: SPACE," Proceedings of The European Navigation Conference, Munich, Germany, July 2005.

89. S. Haykin, "Cognitive Radar Networks," Proceedings of the IEEE International Workshop on Computational Advances in Multi-Sensor Adaptive Processing, December 2005, pp. 1-3.

90. C. Sinka and J. Bito, "Rain Attenuation Countermeasure Technique for Broadband Fixed Wireless Access Networks," Proceedings of the Twelfth International Conference on Antennas and Propagation, 1, UK, March 2003, pp. 441-444.

91. "Environmental Monitoring and Modeling Using Large-Scale Sensor Networks," 2009; available: www.teo.unt.edu.

92. R. Bakker, T. Kirubarajan, B. Currie, and S. Haykin, “Adaptive Radar Detection: A Bayesian Approach," Proceedings of the EPSRC IEE Workshop Nonlinear Non-Gaussian Signal Processing, Peebles, Scotland, July 2002.

93. J. Taylor, Ultra-Wideband Radar Technology, Boca Raton, CRC Press, 2001. 
94. H. Arslan and M. Sahin, UWB-Based Cognitive Radio Networks, Cognitive Wireless Communications Network, New York, SpringerLink, 2007.

95. D. Cabric, S. Mishra, and R. Brodersen, "Implementation Issues in Spectrum Sensing for Cognitive Radios," The ThirtyEighth Asilomar Conference on Signals, Systems and Computers, 1, 2004.

96. Q. Zhao and B. Sadler, "A Survey of Dynamic Spectrum Access," IEEE Signal Processing Magazine, 24, 3, 2007, pp. 79. 89.

97. H. Yamaguchi, "Active Interference Cancellation Technique for MB-OFDM Cognitive Radio," 34th European Microwave Conference, 2, 2004.

98. H. Zhang, X. Zhou, K. Yazdandoost, and I. Chlamtac, "Multiple Signal Waveforms Adaptation in Cognitive Ultra-Wideband Radio Evolution," IEEE Journal on Selected Areas in Communications, 24, 4, April 2006, pp. 878-884.

99. N. Choi, J. Hwang, G. Zheng, N. Han, and J. Kim, "A Cognitive UWB Testbed Employing Adaptive Pulse Generation," 3rd International Conference on Cognitive Radio Oriented Wireless Networks and Communications, Singapore, May 2008, pp. 1-6.

100. "Emergency Ultrawideband Radio for Positioning and Communications," available: http://www.ist-europcom.org.

101. V. Pammer and K. Witrisal, "Ultra Wideband Communication System as Sensor Technology for 3D Mapping," Proceedings of the IEEE International Symposium on Personal, Indoor, Mobile Communications, Helsinki, Finland, September 2006, pp. 1-5.

102. T. A. Weiss and F. K. Jondral, "Spectrum Pooling: An Innovative Strategy for the Enhancement of Spectrum Efficiency," IEEE Communications Magazine, 42, 3, March 2004, pp. 8-14.

103. S. Brandes, I. Cosovic, and M. Schnell, "Reduction of Out-ofBand Radiation in OFDM Based Overlay Systems," IEEE Communications Letters, 10, 6, June 2006, pp. 420-422.

104. H. Mahmoud and H. Arslan, "Sidelobe Suppression in OFDM-Based Spectrum Sharing Systems Using Adaptive Symbol Transition," IEEE Communications Letters, 12, 2, February 2008, pp. 133-135.

105. S. Venkatesh and R. M. Buehrer, "Power Control in UWB Position-Location Networks," Proceedings of the IEEE International Conference on Communications, 9, Istanbul, Turkey, June 2006, pp. 3953-3959.

106. I. Guvenc, H. Arslan, S. Gezici, and H. Kobayashi, "Adaptation of Two Types of Processing Gains for UWB-IR Wireless Sensor Networks," IET Proceedings on Communications, 1, 5, December 2007, pp. 1280-1288.

107. I. Guvenc, Z. Sahinoglu, and P. Orlik, "TOA Estimation for IR-UWB Systems with Different Transceiver Types," IEEE Transactions on Microwave Theory and Techniques, 54, 4, April 2006, pp. 1876-1886.

108. I. Guvenc and Z. Sahinoglu, "Threshold Selection for UWB TOA Estimation Based on Kurtosis Analysis," IEEE Communications Letters, 9, 12, December 2005, pp. 1025-1027.
109. I. Guvenc and Z. Sahinoglu, "Threshold-Based TOA Estimation for Impulse Radio UWB Systems," Proceedings of the IEEE International Conference on Ultra-wideband, Zurich, Switzerland, September 2005, pp. 420-425.

110. Y. Qi, H. Kobayashi, and H. Suda, "On Time-of-Arrival Positioning in a Multipath Environment," IEEE Transactions on Vehicular Technology, 55, 5, September 2006, pp. 1516-1526.

111. S. Gezici, "A Survey on Wireless Position Estimation," Wireless Personal Communications, 44, 3, February 2008, pp. 263-282.

112. N. Devroye and V. Tarokh, "On the Degrees of Freedom in Cognitive Radio Channels," Arxiv preprint arXiv:0707.1859, 2007.

113. N. Devroye, P. Mitran, and V. Tarokh, "Achievable Rates in Cognitive Radio Channels," IEEE Transactions on Information Theory, 52, 5, 2006, pp. 1813-1827.

114. T. Clancy, "Adaptation Overhead in Time-Varying Cognitive Radio Channels," IEEE Consumer Communications and Networking Conference, Las Vegas, Nevada, USA, January 2008, pp. 1009-1013.

115. A. Zeira and P. M. Schultheis, "Time Delay Estimation for Closely Spaced Echoes," Proceedings of the IEEE International Conference on Acoustic, Speech, Signal Processing, Albuquerque, NM, April 1990, pp. 2763-2766.

116. S. Gezici, Z. Tian, G. B. Giannakis, H. Kobayashi, A. F. Molisch, H. V. Poor, and Z. Sahinoglu, "Localization via UltraWideband Radios: A Look at Positioning Aspects for Future Sensor Networks," IEEE Signal Processing Magazine, 22, 4, July 2005, pp. 70-84.

117. B. Alavi and K. Pahlavan, "Studying the Effect of Bandwidth on Performance of UWB Positioning Systems," Proceedings of the IEEE Wireless Communications and Networking Conference, Las Vegas, NV, 2, April 2006, pp. 884-889.

118. C. C. Chong, F. Watanabe, and M. Z. Win, "Effect of Bandwidth on UWB Ranging Error," Proceedings of the IEEE Wireless Communications and Networking Conference, Hong Kong, March 2007, pp. 1559-1564.

119. J. J. Caffery and G. L. Stuber, "Overview of Radiolocation in CDMA Cellular Systems," IEEE Communications Magazine, 36, 4, April 1998, pp. 38-45.

120. I. Guvenc, C. C. Chong, and F. Watanabe, "NLOS Identification and Weighted Least Squares Localization for UWB Systems Using Multipath Channel Statistics," EURASIP Journal on Advances in Signal Processing, 2008, Article ID 271984, 2008, 14 pages.

121. Z. Li, W. Trappe, Y. Zhang, and B. Nath, "Robust Statistical Methods for Securing Wireless Localization in Sensor Networks," Proceedings of the IEEE International Symposium on Information Processing in Sensor Networks, Los Angeles, CA, April 2005, pp. 91-98.

122. P. C. Chen, "A Non-Line-of-Sight Error Mitigation Algorithm in Location Estimation," Proceedings of the IEEE International Conference on Wireless Communications Networking, 1, New Orleans, LA, September 1999, pp. 316-320. 
123. S. Venkatesh and R. M. Buehrer, "A Linear Programming Approach to NLOS Error Mitigation in Sensor Networks," Proceedings of the IEEE International Conference on Information Processing in Sensor Networks, Nashville, TN, April 2006, pp. 301-308.

124. I. Guvenc and C. C. Chong, "A Survey on TOA Based Wireless Localization and NLOS Mitigation Techniques," IEEE Communications Surveys and Tutorials, 11, 3, Third Quarter 2009, pp. 107-124.

125. Y. T. Chan, W. Y. Tsui, H. C. So, and P. C. Ching, "Time of Arrival Based Localization Under NLOS Conditions," IEEE Transactions on Vehicular Technology, 55, 1, January 2006, pp. 17-24.

126. C. Nerguizian, C. Despins, and S. Affes, "Geolocation in Mines with an Impulse Response Fingerprinting Technique and Neural Networks," IEEE Transactions on Wireless Communications, 5, 3, March 2006, pp. 603-611.

127. M. McGuire, K. N. Plataniotis, and A. N. Venetsanopoulos, "Location of Mobile Terminals Using Time Measurements and Survey Points," IEEE Transactions on Vehicular Technology, 52, 4, July 2003, pp. 999-1011.

128. S. Gezici, H. Kobayashi, and H. V. Poor, "A New Approach to Mobile Position Tracking," Proceedings of the IEEE Sarnoff Symposium On Advances in Wired And Wireless Communications, Ewing, NJ, March 2003, pp. 204-207.

129. T.-N. Lin and P.-C. Lin, "Performance Comparison of Indoor Positioning Techniques Based on Location Fingerprinting in Wireless Networks," Proceedings of the International Conference on Wireless Networks, Communications and Mobile Computing, 2, June 2005, pp. 1569-1574.

130. J. Kwon, B. Dundar, and P. Varaiya, "Hybrid Algorithm for Indoor Positioning Using Wireless LAN," Proceedings of the IEEE Vehicular Technology Conference, 7, Los Angeles, CA, September 2004, pp. 4625-4629.

131. R. O. Duda, P. E. Hart, and D. G. Stork, Pattern Classification, Second Edition, New York, Wiley-Interscience, 2000.

132. W. Kim, J. G. Lee, and G. I. Jee, "The Interior-Point Method for an Optimal Treatment of Bias in Trilateration Location," IEEE Transactions on Vehicular Technology, 55, 4, July 2006, pp. 12911301.

133. J. J. Caffery, "A New Approach to the Geometry of TOA Location," Proceedings of the IEEE Vehicular Technology Conference, 4, Boston, MA, September 2000, pp. 1943-1949.

134. V. Pammer and K. Witrisal, "Enhancements to Linear Least Squares Localization through Reference Selection and ML Estimation," Proceedings of the IEEE Wireless Communications and Networking Conference, Las Vegas, NV, March 2008, pp. 284289.

135. Z. Sahinoglu and I. Guvenc, "Multiuser Interference Mitigation in Noncoherent UWB Ranging via Nonlinear Filtering," EURASIP Journal on Wireless Communications and Networking, Article ID 56849, 2006, 10 pages.

136. D. Dardari, A. Giorgetti, and M. Win, "Time-of-Arrival Estimation in the Presence of Narrow and Wide Bandwidth Interfer- ence in UWB Channels," IEEE International Conference on Ultrawideband, Marina Bay, Singapore, September 2007.

137. S. Gezici, H. Celebi, H. Arslan, and H. V. Poor, "Theoretical Limits on Time Delay Estimation for Ultra-Wideband Cognitive Radios," IEEE International Conference on Ultra-wideband, 2, Hanover, Germany, September 2008, pp. 177-180.

138. N. Devroye, P. Mitran, and V. Tarokh, "Limits on Communications in a Cognitive Radio Channel," IEEE Communications Magazine, 44, 6, June 2006, pp. 44-49.

139. P. Mahonen, M. Petrova, and J. Riihijarvi, "Applications of Topology Information for Cognitive Radios and Networks," Proceedings of the IEEE Symposium on New Frontiers in Dynamic Spectrum Access Networks, Dublin, Ireland, April 2007, pp. 103114.

\section{Introducing the Feature Article Authors}

Hasari Celebi received his BSc in the Electronics and Communications Engineering Department from Yildiz Technical University in Istanbul, Turkey, in June 2000, and his MSc in the Electrical Engineering Department from San Jose State University in San Jose, California, USA in May 2004. He received his PhD from the Electrical Engineering Department at the University of South Florida in Tampa, Florida, USA, in August 2008. He is currently a Postdoctoral Research Associate in Electrical and Computer Engineering Department at Texas A\&M University at Qatar.

Dr. Celebi was the recipient of the best paper award at the Fourth International Conference on Cognitive Radio Oriented Wireless Networks and Communications (Crowncom) 2009. He was also the recipient of the Outstanding Graduate Achievement Award at University of South Florida in 2008. Dr. Celebi is a member of the IEEE. His research areas include detection and estimation theory, statistical signal processing, diversity combining, channel propagation modeling, cognitive radio and software defined radio (SDR), cognitive positioning systems, localization, and UWB systems.

Ismail Guvenc received his BS from Bilkent University, Turkey, in 2001; his MS from the University of New Mexico, Albuquerque, NM, in 2003; and the PhD from the University of South Florida, Tampa, FL, in 2006 (with an Outstanding Dissertation Award from the USF Graduate School); all in Electrical Engineering. He was with Mitsubishi Electric Research Labs in Cambridge, MA, in 2005. Since June 2006, he has been with DOCOMO USA Labs, Palo Alto, CA, working as a research engineer. His recent research interests include femtocells, LTE systems, cognitive radio, and UWB communications and localization. He has published more than 50 international conference and journal papers, and several standardization contributions for the IEEE 802.15 and IEEE 802.16 standards. Dr. Guvenc has served on the organizing and technical program committees of several international conferences, and coauthored a book on ultra-wideband position estimation. He has over 15 pending US patent applications, and he is a member of the IEEE.

Sinan Gezici received his BS from Bilkent University, Turkey in 2001, and a PhD in Electrical Engineering from Princeton University in 2006. From April 2006 to January 2007, he worked 
as a Visiting Member of Technical Staff at Mitsubishi Electric Research Laboratories, Cambridge, MA. Since February 2007, he has been an Assistant Professor in the Department of Electrical and Electronics Engineering at Bilkent University. Dr. Gezici's research interests are in the areas of signal detection, estimation, and optimization theory, and their applications to wireless communications and localization systems. Among his publications in these areas is the recent book Ultra-Wideband Positioning Systems: Theoretical Limits, Ranging Algorithms, and Protocols (Cambridge University Press, 2008). Dr. Gezici is a member of the IEEE.

Huseyin Arslan received his $\mathrm{PhD}$ degree in 1998 from Southern Methodist University (SMU), Dallas, TX. From January 1998 to August 2002, he was with the research group of Ericsson Inc., NC, USA, where he was involved with several projects related to $2 \mathrm{G}$ and $3 \mathrm{G}$ wireless cellular communication systems. Since August 2002, he has been with the Electrical Engineering Department of the University of South Florida. In addition, he has worked as part-time consultant for various companies and institutions, including Anritsu Company, the Scientific and Technological Research Council of Turkey - TUBITAK, Lecroy, and XG technologies.

Dr. Arslan's research interests are related to advanced signalprocessing techniques at the physical layer, with cross-layer design for networking adaptivity and quality of service (QoS) control. He is interested in many forms of wireless technologies, including cellular, wireless PAN/LAN/MANs, fixed wireless access, and specialized wireless data networks, such as wireless sensors networks and wireless telemetry. The current research interests are on UWB, OFDM-based wireless technologies with emphasis on WIMAX and IMT-Advanced, and cognitive and software-defined radio. He has served as technical program committee chair, technical program committee member, session and symposium organizer, and workshop chair for several IEEE conferences. He is a member of the editorial board for Wireless Communication and Mobile Computing Journal and Research Letters in Communications. Dr. Arslan is a senior member of the IEEE. $\left.14^{4}\right)$ 